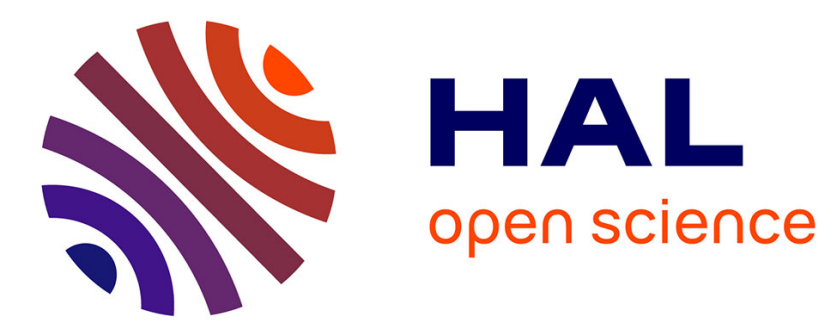

\title{
Geometric analysis for the metropolis algorithm on Lipschitz domains
}

Persi Diaconis, Gilles Lebeau, Laurent Michel

\section{To cite this version:}

Persi Diaconis, Gilles Lebeau, Laurent Michel. Geometric analysis for the metropolis algorithm on Lipschitz domains. Inventiones Mathematicae, 2011, 185 (2), pp.239-281. 10.1007/s00222-0100303-6 . hal-01343544

\author{
HAL Id: hal-01343544 \\ https://hal.science/hal-01343544
}

Submitted on 8 Jul 2016

HAL is a multi-disciplinary open access archive for the deposit and dissemination of scientific research documents, whether they are published or not. The documents may come from teaching and research institutions in France or abroad, or from public or private research centers.
L'archive ouverte pluridisciplinaire HAL, est destinée au dépôt et à la diffusion de documents scientifiques de niveau recherche, publiés ou non, émanant des établissements d'enseignement et de recherche français ou étrangers, des laboratoires publics ou privés. 


\title{
Geometric Analysis for the Metropolis Algorithm on Lipshitz Domains
}

\author{
Persi Diaconis, Gilles Lebeau, Laurent Michel \\ Département de Mathématiques, \\ Université de Nice Sophia-Antipolis \\ Parc Valrose 06108 Nice Cedex 02, France \\ lebeau@unice.fr \\ January 16, 2013
}

\begin{abstract}
This paper gives geometric tools: comparison, Nash and Sobolev inequalities for pieces of the relevent Markov operators, that give useful bounds on rates of convergence for the Metropolis algorithm. As an example, we treat the random placement of $N$ hard discs in the unit square, the original application of the Metropolis algorithm.
\end{abstract}

\section{Contents}

1 Introduction and results 2

2 A proof of theorem $\frac{\operatorname{thm} 1}{1.1}$

3 A proof of theorem $\frac{\operatorname{thm} 2}{1.2} \quad 15$

4 Application to random placement of non-overlapping balls 19

$\begin{array}{ll}\text { References } & 31\end{array}$ 


\section{Introduction and results}

Let $\Omega$ be a bounded, connected subset of $\mathbb{R}^{d}$. We assume that its boundary, $\partial \Omega$ has Lipschitz regularity. Let $B_{1}$ be the unit ball of $\mathbb{R}^{d}$ and $\varphi(z)=\frac{1}{\operatorname{Vol}\left(B_{1}\right)} 1_{B_{1}}(z)$ so that $\int \varphi(z) d z=1$. Let $\rho(x)$ be a measurable positive bounded function on $\bar{\Omega}$ such that $\int_{\Omega} \rho(x) d x=1$. For $\left.\left.h \in\right] 0,1\right]$, set

$$
K_{h, \rho}(x, y)=h^{-d} \varphi\left(\frac{x-y}{h}\right) \min \left(\frac{\rho(y)}{\rho(x)}, 1\right)
$$

and let $T_{h, \rho}$ be the () Metropolis operator associated to these data, that is

$$
\begin{aligned}
& T_{h, \rho}(u)(x)=m_{h, \rho}(x) u(x)+\int_{\Omega} K_{h, \rho}(x, y) u(y) d y \\
& m_{h, \rho}(x)=1-\int_{\Omega} K_{h, \rho}(x, y) d y \geq 0
\end{aligned}
$$

Then the Metropolis kernel $T_{h, \rho}(x, d y)=m_{h, \rho}(x) \delta_{x=y}+K_{h, \rho}(x, y) d y$ is a Markov kernel, the operator $T_{h, \rho}$ is self-adjoint on $L^{2}(\Omega, \rho(x) d x)$, and thus the probability measure $\rho(x) d x$ on $\Omega$ is stationary. For $n \geq 1$, we denote by $T_{h, \rho}^{n}(x, d y)$ the kernel of the iterate operator $\left(T_{h, \rho}\right)^{n}$. For any $x \in \Omega, T_{h, \rho}^{n}(x, d y)$ is a probability measure on $\Omega$, and our main goal is to get some estimates on the rate of convergence, when $n \rightarrow+\infty$, of the probability $T_{h, \rho}^{n}(x, d y)$ toward the stationary probability $\rho(y) d y$.

A good example to keep in mind is the random placement of $N$ non-overlapping discs of radius $\varepsilon>0$ in the unit square. This was the original motivation for the work of Metropolis et al $\left(\left[\mathbb{R K R}^{+} 53\right]\right)$. One version of their algorithm goes as follows: from a feasable configuration, pick a disc (uniformly at random) and a point within distance $h$ of the center of the chosen disc (uniformly at random). If recentering the chosen disc at the chosen point results in a feasable configuration, the change is made. Otherwise, the configuration is kept as it started. If $N$ is fixed and $\varepsilon$ and $h$ are small, this gives a Markov chain with a uniform stationary distribution over all feasable configurations. The state space consists of the $N$ centers corresponding to feasible configurations. It is a bounded domain with a Lipshitz boundary (see section $\frac{\text { sect }}{4,}$ proposition $\frac{\text { prop }}{4.1 \text { ). It is }}$ non-convex (because of the non-overlaping constraints). The scientific motivation for the study of random packing of hard discs is clearly described in Uhlenbeck ([?], section 5, pg 18). An overview of the large literature is in Lowen (fow . Entry to the zoo of modern algorithms to do the simulation (particularly in the dense case) with many examples is in [German guy, reference coming]. Further discussion, showing that the problem is still of current interest, is in Radin ([?]).

We shall denote by $g(h, \rho)$ the spectral gap of the Metropolis operator $T_{h, \rho}$. It is defined as the best constant such that the following inequality holds true for all $u \in$ $L^{2}(\rho)=L^{2}(\Omega, \rho(x) d x)$

$$
\|u\|_{L^{2}(\rho)}^{2}-(u \mid 1)_{L^{2}(\rho)}^{2} \leq \frac{1}{g(h, \rho)}\left(u-T_{h, \rho} u \mid u\right)_{L^{2}(\rho)}
$$

or equivalently

$$
\int_{\Omega \times \Omega}|u(x)-u(y)|^{2} \rho(x) \rho(y) d x d y \leq \frac{1}{g(h, \rho)} \int_{\Omega \times \Omega} K_{h, \rho}(x, y)|u(x)-u(y)|^{2} \rho(x) d x d y
$$


def1 Definition 1.1 We say that an open set $\Omega \subset \mathbb{R}^{d}$ is Lipschitz if it is bounded and for all $a \in \partial \Omega$ there exists an orthonormal basis $\mathcal{R}_{a}$ of $\mathbb{R}^{d}$, an open set $\left.V=V^{\prime} \times\right]-\alpha, \alpha[$ and $a$ Lipschitz map $\left.\eta: V^{\prime} \rightarrow\right]-\alpha, \alpha\left[\right.$ such that in the coordinates of $\mathcal{R}_{a}$, we have

$$
\begin{aligned}
& V \cap \Omega=\left\{\left(y^{\prime}, y_{d}<\eta\left(y^{\prime}\right)\right),\left(y^{\prime}, y_{d}\right) \in V^{\prime} \times\right]-\alpha, \alpha[\} \\
& V \cap \partial \Omega=\left\{\left(y^{\prime}, \eta\left(y^{\prime}\right)\right), y^{\prime} \in V^{\prime}\right\} .
\end{aligned}
$$

Our first result is the following:

thm1 Theorem 1.1 Let $\Omega$ be an open, connected, bounded and Lipschitz subset of $\mathbb{R}^{d}$. Let $0<m \leq M<\infty$ be given numbers. There exists $\left.h_{0}>0, \delta_{0} \in\right] 0,1 / 2[$ and constants $C_{i}>0$ such that for any $\left.\left.h \in\right] 0, h_{0}\right]$, and any probability density $\rho$ on $\Omega$ which satisfies for all $x, m \leq \rho(x) \leq M$, the following holds true.

i) The spectrum of $T_{h, \rho}$ is a subset of $\left[-1+\delta_{0}, 1\right], 1$ is a simple eigenvalue of $T_{h, \rho}$, and $\operatorname{Spec}\left(T_{h, \rho}\right) \cap\left[1-\delta_{0}, 1\right]$ is discrete. Moreover, for any $0 \leq \lambda \leq \delta_{0} h^{-2}$, the number of eigenvalues of $T_{h, \rho}$ in $\left[1-h^{2} \lambda, 1\right]$ (with multiplicity) is bounded by $C_{1}(1+\lambda)^{d / 2}$.

ii) The spectral gap satisfies

$$
C_{2} h^{2} \leq g(h, \rho) \leq C_{3} h^{2}
$$

and the following estimate holds true for all integer $n$

$$
\sup _{x \in \Omega}\left\|T_{h, \rho}^{n}(x, d y)-\rho(y) d y\right\|_{T V} \leq C_{4} e^{-n g(h, \rho)}
$$

The next result will give some more information on the behavior of the spectral gap $g(h, \rho)$ when $h \rightarrow 0$. To state this result, let

$$
\alpha_{d}=\int \varphi(z) z_{1}^{2} d z=\frac{1}{d} \int \varphi(z)|z|^{2} d z=\frac{1}{d+2}
$$

and let us define $\nu(\rho)$ as the best constant such that the following inequality holds true for all $u$ in the Sobolev space $H^{1}(\Omega)$

$$
\|u\|_{L^{2}(\rho)}^{2}-(u \mid 1)_{L^{2}(\rho)}^{2} \leq \frac{1}{\nu(\rho)} \frac{\alpha_{d}}{2} \int_{\Omega}|\nabla u|^{2}(x) \rho(x) d x
$$

or equivalently

$$
\int_{\Omega \times \Omega}|u(x)-u(y)|^{2} \rho(x) \rho(y) d x d y \leq \frac{\alpha_{d}}{\nu(\rho)} \int_{\Omega}|\nabla u|^{2}(x) \rho(x) d x
$$

Observe that for a Lipschitz domain $\Omega$, the constant $\nu(\rho)$ is well-defined thanks to Sobolev embedding. For a smooth density $\rho$, this number $\nu(\rho)>0$ is strongly related to the unbounded operator $L_{\rho}$ acting on on $L^{2}(\rho)$

$$
\begin{aligned}
& L_{\rho}(u)(x)=\frac{-\alpha_{d}}{2}\left(\Delta u+\frac{\nabla \rho}{\rho} \cdot \nabla u\right) \\
& D\left(L_{\rho}\right)=\left\{u \in H^{1}(\Omega),-\Delta u \in L^{2}(\Omega),\left.\partial_{n} u\right|_{\partial \Omega}=0\right\}
\end{aligned}
$$


When $\Omega$ has smooth boundary, standard elliptic regularity results show that for any $u \in H^{1}(\Omega)$ such that $-\Delta u \in L^{2}(\Omega)$, the normal derivative of $u$ at the boundary, $\partial_{n} u=$ $\left.\vec{n}(x) \cdot \nabla u\right|_{\partial \Omega}$ is well define and belongs to the Sobolev space $H^{1 / 2}(\Omega)$. Here, we denote by $\vec{n}(x)$ the incoming unitary normal vector to $\partial \Omega$ at a point $x$. In the case where $\partial \Omega$ has only Lipschitz regularity, the normal $\vec{n}(x)$ is well defined for allmost every $x \in \partial \Omega$ (with respect to the measure $\sigma$ induced on the boundary). Using a suitable covering of $\Omega$ it is possible to define a trace operator $\gamma_{0}: H^{1}(\Omega) \mapsto L^{2}(\partial \Omega)$ which is equal to the usual trace in the case of a smooth boundary. We sometimes denote $\gamma_{0}(u)=\left.u\right|_{\partial \Omega}$. The space defined by $H^{1 / 2}(\partial \Omega)=\operatorname{Ran}\left(\gamma_{0}\right)$ doesn't depend on the charts used to define $\gamma_{0}$, and equipped with the norm $\|u\|_{H^{1 / 2}}=\inf \left\{\|v\|_{H^{1}}, \gamma_{0}(v)=u\right\}$ it is a reflexif Banach space. Then, we can set $H^{-1 / 2}(\partial \Omega)=H^{1 / 2}(\partial \Omega)^{*}$ and for $u \in H^{-1 / 2}(\partial \Omega)$, the support of $u$ can be defined in a standard way. The trace operator acting on vector fields $u \in L^{2}, \operatorname{div}(u) \in L^{2}$

$$
\gamma_{1}:\left\{u \in\left(L^{2}(\Omega)\right)^{d}, \operatorname{div}(u) \in L^{2}(\Omega)\right\} \rightarrow H^{-1 / 2}(\partial \Omega)
$$

is then defined by the formula

$$
\int_{\Omega} \operatorname{div}(u)(x) v(x) d x=-\int_{\Omega} u(x) \cdot \nabla v(x) d x-\left.\int_{\partial \Omega} \gamma_{1}(u) v\right|_{\partial \Omega} d \sigma(x)
$$

In particular, for $u \in H^{1}(\Omega)$ satisfying $-\Delta u=\operatorname{div} \nabla u \in L^{2}(\Omega)_{11.3}$ we can define $\left.\partial_{n} u\right|_{\partial \Omega}=$ $\gamma_{1}(\nabla u) \in H^{-1 / 2}(\partial \Omega)$ and the set $D\left(L_{\rho}\right)$ is well defined. From (11.3.3) we deduce that for any $u \in H^{1}(\Omega)$ with $\Delta u \in L^{2}$ and any $v \in H^{1}(\Omega)$ we have

$$
\left\langle\left(L_{\rho}+1\right) u, v\right\rangle_{L^{2}(\rho)}=\frac{\alpha_{d}}{2}\left(\langle\nabla u, \nabla v\rangle_{L^{2}(\rho)}+\left\langle\partial_{n} u, \rho v\right\rangle_{H^{-1 / 2}(\partial \Omega), H^{1 / 2}(\partial \Omega)}\right)+\langle u, v\rangle_{L^{2}(\rho)}
$$

Then, it is standard that $L_{\rho}$ is the self-adjoint realisation of the Dirichlet form

$$
\frac{\alpha_{d}}{2} \int_{\Omega}|\nabla u(x)|^{2} \rho(x) d x
$$

Sobolev embeddings show that $L_{\rho}$ has a compact resolvant and we denote its spectrum by $\nu_{0}=0<\nu_{1}<\nu_{2}<\ldots$ and by $m_{j}$ the multiplicity of $\nu_{j}$. In particular, $\nu(\rho)=\nu_{1}$. Observe also that $m_{0}=1$ since $\operatorname{Ker} L$ is spaned by the constant function equal to 1 .

To state our theorem, we need

def2 Definition 1.2 Let $\Omega$ be a Lipschitz open set of $\mathbb{R}^{d}$. We say that $\partial \Omega$ is stratified if $\partial \Omega=\Gamma_{\text {reg }} \cup \Gamma_{\text {sing }}, \Gamma_{\text {reg }} \cap \Gamma_{\text {sing }}=\emptyset$ with $\Gamma_{\text {reg }}$ reunion of smooth hypersurfaces, relatively open in $\partial \Omega$, and $\Gamma_{\text {sing }}$ a closed subset of $\mathbb{R}^{d}$ such that

$$
v \in H^{-1 / 2}(\partial \Omega) \text { and } \operatorname{support}(v) \subset \Gamma_{\text {sing }} \Longrightarrow v=0
$$

Observe that $\frac{u n i g}{1.16}$ is obviously satisfied if $\partial \Omega$ is smooth, since in that case one can take $\Gamma_{\text {sing }}=\emptyset$. More generally, if $\Omega$ is a Lipschitz open set of $\mathbb{R}^{d}$ such that $\partial \Omega=$ $\Gamma_{\text {reg }} \cup \Gamma_{\text {sing }}, \Gamma_{\text {reg }} \cap \Gamma_{\text {sing }}=\emptyset$, where $\Gamma_{\text {reg }}$ is a smooth hypersurface of $\mathbb{R}^{d}$, relatively open in $\partial \Omega$, and $\Gamma_{\text {sing }}$ a closed subset of $\mathbb{R}^{d}$ such that $\Gamma_{\text {sing }}=\cup_{j \geq 2} S_{j}$ where the $S_{j}$ are smooth disjoints submanifolds of $\mathbb{R}^{d}$ such that

$$
\operatorname{codim}_{\mathbb{R}^{d}} S_{j} \geq j, \quad \cup_{k \geq j} S_{k}=\overline{S_{j}}
$$


then $\Omega$ is stratified, since in that case, if $v \in H^{-1 / 2}(\partial \Omega)$ is such that near a point $x_{0}$, the support of $v$ is contained in a submanifold $S$ of codimension $\geq 2$ in $\mathbb{R}^{d}$, then $v=0$ near $x_{0}$. This follows from the fact that $S$ has codimension $\geq 1$ in $\partial \Omega$, and if $u \in \mathcal{D}^{\prime}\left(\mathbb{R}^{p}\right)$ is such that $u \in H_{l o c}^{-1 / 2}\left(\mathbb{R}^{p}\right)$ and $\operatorname{support}(u) \subset\left\{x_{1}=0\right\}$, then $u=0$. As an example, a cube in $\mathbb{R}^{d}$ is stratified.

thm2 Theorem 1.2 Let $\Omega$ be an open, connected, bounded and Lipschitz subset of $\mathbb{R}^{d}$, such that $\partial \Omega$ is stratified. Assume that the positive density $\rho$ is continuous on $\bar{\Omega}$. Then

$$
\lim _{h \rightarrow 0} h^{-2} g(h, \rho)=\nu(\rho)
$$

Moreover, if the density $\rho$ is smooth up to the regular part $\Gamma_{\text {reg }}$ of the boundary $\partial \Omega$, then for any $R>0$ and $\varepsilon>0$ such that $\nu_{j+1}-\nu_{j}>2 \varepsilon$ for $\nu_{j+2}<R$, there exists $h_{1}>0$ such that one has for all $\left.h \in] 0, h_{1}\right]$

$$
\left.\left.\operatorname{Spec}\left(\frac{1-T_{h, \rho}}{h^{2}}\right) \cap\right] 0, R\right] \subset \cup_{j \geq 1}\left[\nu_{j}-\varepsilon, \nu_{j}+\varepsilon\right]
$$

and the number of eigenvalues of $\frac{1-T_{h, \rho}}{h^{2}}$ in the interval $\left[\nu_{j}-\varepsilon, \nu_{j}+\varepsilon\right]$ is equal to $m_{j}$.

Theorem $\frac{\operatorname{thm} 1}{1.1 \text { is proved in section } 2 \text {. Thec2 }}$. This is done from the spectrum of the operator by comparison with a 'ball walk' on a big box $B$ containing $\Omega$. One novelty is the use of 'normal extensions' of functions from $\Omega$ to $B$. When the Dirichlet forms and stationary distributions for random walk on a compact group are comparible, the rates of convergence are comparable as well (l? ? $^{3}$ lemma ??). Here, the Metropolis Markov chain is far from a random walk on a group. Indeed, because of the holding implicit in the Metropolis algorithm, the operator doesnt have any smoothing properties. The transfer of information is carried out by a Sobolev inequality for a spectraly truncated part of the operator. This is transfered to a Nash inequality and then an inductive argument of Hebisch (see also[ ]) is used to get decay bounds on iterates of the kernel. A further technique is the use of crude Weyl type estimates to get bounds on the number of eigenvalues close to 1 . All of these enter the proof of the total variation estimate $1_{1}^{1.7} 7$. All of these techniques seem broadly applicable. Theorem $\frac{t h m 2}{1.2}$ is proved in section 3 . It gives rigourous underpinnings to a general picture of the spectrum of the Metropolis algorithm based on small steps. This was observed and proved in special cases ([?], [1]107]). The picture is this: because of the holding (or presence of the multiplier $m_{h, \rho}$ in 1.2 ) in the Metropolis algorithm, the operator always has continuous spectrum. This is well isolated from 1 and can be neglected in bounding rates of convergence. The spectrum near 1 is discrete and for $h$ small, merges with the spectrum of an associated Neumann problem. This is an analytic version of the convergence of the discrete time Metropolis chain to the Langevin diffusion with generator Chen reference coming COAUTHORS SAY A SENTENCE OR TWO ABOUT THE

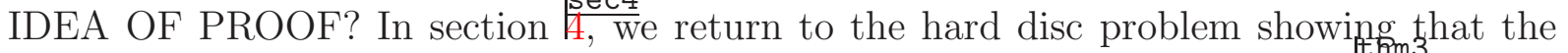
operators and domains involved satisfy our hypothesis ${ }_{\text {thm } 2}$ Precisely, in theorem $\frac{t h m 3}{4.1}$ we shall

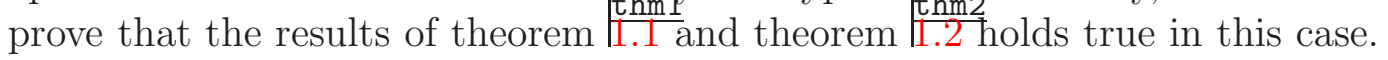

\section{A proof of theorem $\frac{\operatorname{thm} 1}{1.1}$}

Let us recall that

$$
K_{h, \rho}(x, y)=h^{-d} \varphi\left(\frac{x-y}{h}\right) \min \left(\frac{\rho(y)}{\rho(x)}, 1\right)
$$


so that

$$
\begin{aligned}
& T_{h, \rho}(u)=u-Q_{h, \rho}(u) \\
& Q_{h, \rho}(u)(x)=\int_{\Omega} K_{h, \rho}(x, y)(u(x)-u(y)) d y \\
& \left(\left(1-T_{h, \rho}\right) u \mid u\right)_{L^{2}(\rho)}=\frac{1}{2} \iint_{\Omega \times \Omega}|u(x)-u(y)|^{2} K_{h, \rho}(x, y) \rho(x) d x d y
\end{aligned}
$$

For the proof of theorem $\frac{\operatorname{thm} 1}{1.1,}$ we will not really care on the precise choice of the density $\rho$. In fact, if $\rho_{1}, \rho_{2}$ are two densities such that $m \leq \rho_{i}(x) \leq M$ for all $x$, then

$$
\begin{aligned}
& \rho_{2}(x) \leq \rho_{1}(x)\left(1+\frac{\left\|\rho_{1}-\rho_{2}\right\|_{\infty}}{m}\right) \\
& K_{h, \rho_{1}}(x, y) \rho_{1}(x) \leq K_{h, \rho_{2}}(x, y) \rho_{2}(x)\left(1+\frac{\left\|\rho_{1}-\rho_{2}\right\|_{\infty}}{m}\right)
\end{aligned}
$$

and this implies using the definition $\frac{\mid g a p 2}{1.4}$ of the spectral gap and of $\nu_{\rho}$

$$
\begin{aligned}
& \frac{g_{h, \rho_{1}}}{g_{h, \rho_{2}}} \leq\left(1+\frac{\left\|\rho_{1}-\rho_{2}\right\|_{\infty}}{m}\right)^{3} \\
& \frac{\nu_{\rho_{1}}}{\nu_{\rho_{2}}} \leq\left(1+\frac{\left\|\rho_{1}-\rho_{2}\right\|_{\infty}}{m}\right)^{3}
\end{aligned}
$$

In particular, it is sufficient to prove 1 gap3 3 for a constant density.

Observe that since $\Omega$ is Lipschitz, from $\frac{1.2}{1.2}$ and $\frac{\text { comp } 1}{2.3, \text { there exists } h_{0}>0, \delta_{0}>0 \text { such }}$ that for any density $\rho$ with $m \leq \rho(x) \leq M$ one has $\sup _{x \in \Omega} m_{h, \rho}(x) \leq 1-2 \delta_{0}$. Thus the essential spectrum of $T_{h}$ is a subset of $\left[0,1-2 \delta_{0}\right]$. The proof that for some $\delta_{0}>0$, independent of $\rho$, one has $\operatorname{Spec}\left(T_{h, \rho}\right) \subset\left[-1+\delta_{0}, 1\right]$ for all $\left.\left.h \in\right] 0, h_{0}\right]$ is the following: one has

$$
\left(u+T_{h, \rho} u \mid u\right)_{L^{2}(\rho)}=\frac{1}{2} \int_{\Omega \times \Omega} K_{h, \rho}(x, y)|u(x)+u(y)|^{2} \rho(x) d x d y+2\left(m_{h, \rho} u \mid u\right)_{L^{2}(\rho)}
$$

Therefore, it is sufficient to prove that there exists $h_{0}, C_{0}>0$ such that the following inequality holds true for all $\left.h \in] 0, h_{0}\right]$ and all $u \in L^{2}(\Omega)$

$$
\int_{\Omega \times \Omega} h^{-d} \varphi\left(\frac{x-y}{h}\right)|u(x)+u(y)|^{2} d x d y \geq C_{0}\|u\|_{L^{2}(\Omega)}^{2}
$$

Let $\omega_{j} \subset \Omega, \cup_{j} \omega_{j}=\Omega$ be a covering of $\Omega$ such that $\operatorname{diam}\left(\omega_{j}\right)<h$ and for some $C_{i}>0$ independent of $h, \operatorname{vol}\left(\omega_{j}\right) \geq C_{1} h^{d}$, and for any $j$, the number of $k$ such that $\omega_{j} \cap \omega_{k} \neq \emptyset$ is less than $C_{2}$. Such a covering exists as $\Omega$ is Lipschitz. Then

$$
\begin{aligned}
& C_{2} \int_{\Omega \times \Omega} h^{-d} \varphi\left(\frac{x-y}{h}\right)|u(x)+u(y)|^{2} d x d y \geq \sum_{j} \int_{\omega_{j} \times \omega_{j}} h^{-d} \varphi\left(\frac{x-y}{h}\right)|u(x)+u(y)|^{2} d x d y \\
& \geq \sum_{j} h^{-d} \frac{1}{\left|B_{1}\right|} \int_{\omega_{j} \times \omega_{j}}|u(x)+u(y)|^{2} d x d y \geq \sum_{j} 2 h^{-d} \frac{1}{\left|B_{1}\right|} \operatorname{vol}\left(\omega_{j}\right)\|u\|_{L^{2}\left(\omega_{j}\right)}^{2} \geq \frac{2 C_{1}}{\left|B_{1}\right|}\|u\|_{L^{2}(\Omega)}^{2}
\end{aligned}
$$

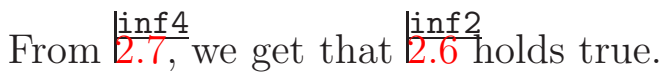

For the proof of (lap3 3 we need a suitable covering of $\Omega$. Given $\epsilon>0$ small enough, there exists some open sets $\Omega_{0}, \ldots, \Omega_{N}$ such that $\left\{x \in \mathbb{R}^{d}\right.$, $\left.\operatorname{dist}(x, \bar{\Omega}) \leq \epsilon^{2}\right\} \subset \cup_{j=0}^{N} \Omega_{j}$, where the $\Omega_{j}$ 's have the following properties: 
1. $\Omega_{0}=\left\{x \in \Omega, d(x, \partial \Omega)>\epsilon^{2}\right\}$

2. For $j=1, \ldots, N$, there exists $r_{j}>0$, an affine isometry $R_{j}$ of $\mathbb{R}^{d}$ and a Lipschitz $\operatorname{map} \varphi_{j}: \mathbb{R}^{d-1} \rightarrow \mathbb{R}$ such that, denoting $\tilde{\phi}_{j}\left(x^{\prime}, x_{d}\right)=\left(x^{\prime}, x_{d}+\varphi_{j}\left(x^{\prime}\right)\right)$ and $\phi_{j}=R_{j} \circ \tilde{\phi}_{j}$, we have

$$
\begin{aligned}
& \left.\phi_{j} \text { is injective on } B\left(0,2 r_{j}\right) \times\right]-2 \epsilon, 2 \epsilon[ \\
& \Omega_{j}=\phi_{j}\left(B\left(0, r_{j}\right) \times\right]-\epsilon, \epsilon[) \\
& \Omega_{j} \cap \Omega=\phi_{j}\left(B\left(0, r_{j}\right) \times\right] 0, \epsilon[) \\
& \phi_{j}\left(B\left(0,2 r_{j}\right) \times\right] 0,2 \epsilon[) \subset \Omega
\end{aligned}
$$

We put our open set $\Omega$ in a large box $B=]-A / 2, A / 2^{[}{ }^{d}$ and for $j=0, \ldots, N$ we let $\chi_{j} \in C_{0}^{\infty}\left(\Omega_{j}\right)$ be such that $\sum_{j} \chi_{j}(x)=1$ for $\operatorname{dist}(x, \bar{\Omega}) \leq \epsilon^{2}$. For any function $u \in L^{2}(\Omega)$, let $u_{j}, j=0, \ldots, N$ be defined in a neighbourhood of $\Omega_{j}$ by $u_{j}=u \circ \phi_{j} \circ S \circ \phi_{j}^{-1}$, where $S\left(x^{\prime}, x_{d}\right)=\left(x^{\prime},-x_{d}\right)$ if $x_{d}<0$ and $S\left(x^{\prime}, x_{d}\right)=\left(x^{\prime}, x_{d}\right)$ if $x_{d} \geq 0$. For $x \in \Omega \cap \Omega_{j}$, one has $u_{j}(x)=u(x)$ and we define

$$
E(u)(x)=\sum_{j=0}^{N} \chi_{j}(x) u_{j}(x)
$$

We observe that $\tilde{\phi}_{j}^{-1}(x)=\left(x^{\prime}, x_{d}-\varphi_{j}\left(x^{\prime}\right)\right)$. Consequently, as $\varphi_{j}$ is Lipschitz-continuous, then $\phi_{j}$ and $\phi_{j}^{-1}$ are also Lipschitz-continuous. Hence, formula (2.3. $)$, gives us an extension map from $L^{2}(\Omega)$ into $L^{2}(B)$, which is also bounded from $H^{1}(\Omega)$ into $H^{1}(B)$. For $u \in L^{2}(\Omega), v \in L^{2}(B)$, set

$$
\begin{aligned}
& \mathcal{E}_{h, \rho}(u)=\left(\left(1-T_{h, \rho}\right) u \mid u\right)_{L^{2}(\rho)} \\
& \mathcal{E}_{h}(v)=\iint_{B \times B,|x-y| \leq h} h^{-d}|v(x)-v(y)|^{2} d x d y
\end{aligned}
$$

Since for $A$ large, $E(u)$ vanishes near the boundary of $B$, we can extend $v=E(u)$ as a A-periodic function on $\mathbb{R}^{d}$, and write its Fourier serie $v(x)=E(u)(x)=\sum_{k \in \mathbb{Z}^{d}} c_{k}(v) e^{2 i \pi k x / A}$ with $c_{k}(v)=A^{-d} \int_{B} e^{-2 i \pi k x / A} v(x) d x$. Then

$$
\begin{aligned}
& \|E(u)\|_{L^{2}(B)}^{2}=A^{d} \sum_{k}\left|c_{k}\right|^{2} \simeq\|u\|_{L^{2}(\Omega)}^{2} \\
& \|E(u)\|_{H^{1}(B)}^{2}=A^{d} \sum_{k}\left(1+4 \pi^{2} k^{2} / A^{2}\right)\left|c_{k}\right|^{2} \simeq\|u\|_{H^{1}(\Omega)}^{2}
\end{aligned}
$$

Moreover, one gets

$$
\begin{aligned}
& \mathcal{E}_{h}(v)=A^{d} \sum_{k}\left|c_{k}\right|^{2} \theta(h k) \\
& \theta(\xi)=\int_{|z| \leq 1}\left|e^{2 i \pi \xi z / A}-1\right|^{2} d z
\end{aligned}
$$

Observe that the function $\theta$ is non-negative, quadratic near 0 and has a positive lower bound for $|\xi| \geq 1$.

lem0 Lemma 2.1 For all $\alpha>1$, there exists $C>0$ and $h_{0}>0$ such that

$$
\left.\left.\forall u \in L^{2}(\Omega), \forall h \in\right] 0, h_{0}\right], \mathcal{E}_{\alpha h, \rho}(u) \leq C \mathcal{E}_{h, \rho}(u) .
$$




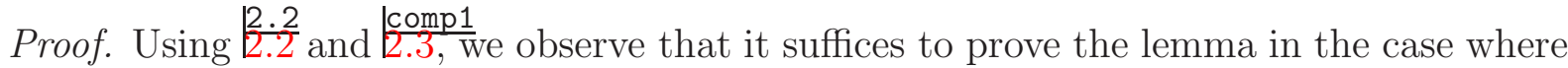
$\rho(x)=\rho$ is constant, and we first we show the result when $\Omega$ is convex. In that case, since $|u(x)-u(y)| \leq\left|u(x)-u\left(\frac{x+y}{2}\right)\right|+\left|u\left(\frac{x+y}{2}\right)-u(y)\right|$, one has

$$
\begin{aligned}
\mathcal{E}_{\alpha h, \rho}(u) & =\frac{(h \alpha)^{-d}}{2 \operatorname{Vol}\left(B_{1}\right)} \int_{\Omega} \int_{\Omega} 1_{|x-y| \leq \alpha h}|u(x)-u(y)|^{2} \rho d x d y \\
& \leq \frac{(h \alpha)^{-d}}{\operatorname{Vol}\left(B_{1}\right)} \int_{\Omega} \int_{\Omega} 1_{|x-y| \leq \alpha h}\left|u(x)-u\left(\frac{x+y}{2}\right)\right|^{2} \rho d x d y \\
& \leq \frac{2^{2-d}(h \alpha / 2)^{-d}}{V o l\left(B_{1}\right)} \int_{\phi(\Omega \times \Omega)} 1_{|x-y| \leq \frac{\alpha h}{2}}|u(x)-u(y)|^{2} \rho d x d y
\end{aligned}
$$

where $\phi(x, y)=\left(x, \frac{x+y}{2}\right)$. As $\Omega$ is convex $\phi(\Omega \times \Omega) \subset \Omega \times \Omega$ and we get $\mathcal{E}_{\alpha h, \rho}(u) \leq 2 \mathcal{E}_{\frac{\alpha h}{2}, \rho}(u)$. Iterating this process we obtain the anounced result for convex domains.

In the general case, we use the local covering introduced in 2.8 . Let $\Omega_{i}^{+}=\Omega_{i} \cap \Omega$ (respectively $\Omega_{i}^{-}=\Omega_{i} \cap\left(\mathbb{R}^{d} \backslash \Omega\right)$ ) and $U_{i}(h)=\left\{(x, y) \in \Omega_{i}^{+} \times \Omega,|x-y| \leq \alpha h\right\}$. Since by 2.2, $\Omega \subset \cup_{i} \Omega_{i}^{+}$, we have $\mathcal{E}_{\alpha h, \rho}(u) \leq \sum_{i=0}^{N} \mathcal{E}_{\alpha h, \rho}^{i}(u)$ with

$$
\mathcal{E}_{\alpha h, \rho}^{i}(u)=\frac{(\alpha h)^{-d}}{2 \operatorname{Vol}\left(B_{1}\right)} \int_{U_{i}(h)} 1_{|x-y| \leq \alpha h}|u(x)-u(y)|^{2} \rho d x d y .
$$

Let us estimate $\mathcal{E}_{\alpha h, \rho}^{0}(u)$. For $\left.h \in\right] 0, \epsilon^{2} / \alpha\left[\right.$ and $(x, y) \in U_{0}(h)$, we have $[x, y] \subset \Omega$. Therefore, the change of variable $\phi(x, y)=\left(x, \frac{x+y}{2}\right)$ maps $U_{0}(h)$ into $\Omega_{0} \times \Omega$ and we get as above

$$
\mathcal{E}_{\alpha h, \rho}^{0}(u) \leq \frac{(\alpha h)^{-d}}{\operatorname{Vol}\left(B_{1}\right)} \int_{U_{0}(h)} 1_{|x-y| \leq \alpha h}\left|u(x)-u\left(\frac{x+y}{2}\right)\right|^{2} \rho d x d y \leq 2 \mathcal{E}_{\frac{\alpha h}{2}, \rho}(u) .
$$

For $i \neq 0$ and $h>0$ small enough, we remark that $U_{i}(h) \subset \tilde{\Omega}_{i}^{+} \times \tilde{\Omega}_{i}^{+}$, where $\tilde{\Omega}_{i}^{ \pm}=$ $\phi_{i}\left(B\left(0,2 r_{i}\right) \times\left\{0< \pm x_{d}<2 \epsilon\right\}\right)$. Denoting $\left.Q_{i}=B\left(0, r_{i}\right) \times\right] 0, \epsilon\left[, \tilde{Q}_{i}=B\left(0,2 r_{i}\right) \times\right] 0,2 \epsilon[$, we can use the Lipschitz-continuous change of variable $\phi_{i}: \tilde{Q}_{i} \rightarrow \tilde{\Omega}_{i}^{+} \subset \Omega$ to get

$$
\mathcal{E}_{\alpha h, \rho}^{i}(u) \leq \frac{(\alpha h)^{-d}}{2 V o l\left(B_{1}\right)} \int_{\tilde{Q}_{i}} \int_{\tilde{Q}_{i}} J_{\phi_{i}}(x) J_{\phi_{i}}(y) 1_{\left|\phi_{i}(x)-\phi_{i}(y)\right| \leq \alpha h}\left|u \circ \phi_{i}(x)-u \circ \phi_{i}(y)\right|^{2} \rho d x d y
$$

where the Jacobian $J_{\phi_{i}}$ of $\phi_{i}$ is a bounded function defined almost everywhere. As both $\phi_{i}, \phi_{i}^{-1}$ are Lipschitz-continuous, there exists $M_{i}, m_{i}>0$ such that for all $x, y \in \tilde{Q}_{i}$ we have $m_{i}|x-y| \leq\left|\phi_{i}(x)-\phi_{i}(y)\right| \leq M_{i}|x-y|$. Therefore,

$$
\mathcal{E}_{\alpha h, \rho}^{i}(u) \leq C h^{-d} \int_{\tilde{Q}_{i}} \int_{\tilde{Q}_{i}} 1_{|x-y| \leq \frac{\alpha h}{m_{i}}}\left|u \circ \phi_{i}(x)-u \circ \phi_{i}(y)\right|^{2} \rho d x d y,
$$

where $C$ denotes a positive constant changing from line to line. As $\tilde{Q}_{i}$ is convex, it follows from the study of the convex case that

$$
\begin{aligned}
\mathcal{E}_{\alpha h, \rho}^{i}(u) & \leq C h^{-d} \int_{\tilde{Q}_{i}} \int_{\tilde{Q}_{i}} 1_{|x-y| \leq \frac{h}{M_{i}}}\left|u \circ \phi_{i}(x)-u \circ \phi_{i}(y)\right|^{2} \rho d x d y \\
& \leq C h^{-d} \int_{\tilde{Q}_{i}} \int_{\tilde{Q}_{i}} 1_{\left|\phi_{i}(x)-\phi_{i}(y)\right| \leq h}\left|u \circ \phi_{i}(x)-u \circ \phi_{i}(y)\right|^{2} d x d y \rho \\
& \leq C h^{-d} \int_{\tilde{\Omega}_{i}^{+}} \int_{\tilde{\Omega}_{i}^{+}} 1_{|x-y| \leq h}|u(x)-u(y)|^{2} \rho d x d y \leq C_{i} \mathcal{E}_{h, \rho}(u)
\end{aligned}
$$


and the proof is complete.

lem1 Lemma 2.2 There exist $C_{0}, h_{0}>0$ such that the following holds true for any $\left.\left.h \in\right] 0, h_{0}\right]$ and any $u \in L^{2}(\rho)$

$$
\mathcal{E}_{h, \rho}(u) / C_{0} \leq \mathcal{E}_{h}(E(u)) \leq C_{0}\left(\mathcal{E}_{h, \rho}(u)+h^{2}\|u\|_{L^{2}}^{2}\right)
$$

As a byproduct, there exists $C_{1}$ such that for all $\left.\left.h \in\right] 0, h_{0}\right]$, any function $u \in L^{2}(\rho)$ such that

$$
\|u\|_{L^{2}(\rho)}^{2}+h^{-2}\left(\left(1-T_{h, \rho}\right) u \mid u\right)_{L^{2}(\rho)} \leq 1
$$

admits a decomposition $u=u_{L}+u_{H}$ with $u_{L} \in H^{1}(\Omega),\left\|u_{L}\right\|_{H^{1}} \leq C_{1}$, and $\left\|u_{H}\right\|_{L^{2}} \leq C_{1} h$.

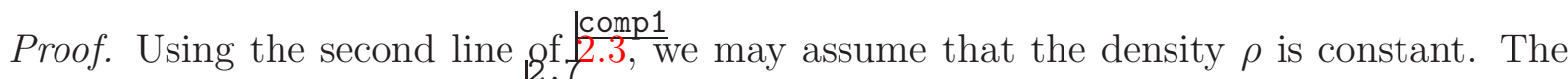
proof of the left inequality in $\frac{2.20}{2}$ is obvious. For the upper bound, we remark that there exists $C>0$ such that $\mathcal{E}_{h}(E(u)) \leq C \sum_{j=0}^{N}\left(\mathcal{E}_{h}^{j, 1}+\mathcal{E}_{h}^{j, 2}\right)$ with

$$
\mathcal{E}_{h}^{j, 1}=h^{-d} \int_{B \times B} 1_{|x-y| \leq h}\left|\chi_{j}(x)-\chi_{j}(y)\right|^{2}\left|u_{j}(x)\right|^{2} d x d y
$$

and

$$
\mathcal{E}_{h}^{j, 2}=h^{-d} \int_{B \times B} 1_{|x-y| \leq h}\left|\chi_{j}(y)\right|^{2}\left|u_{j}(x)-u_{j}(y)\right|^{2} d x d y
$$

As the functions $\chi_{j}$ are regular, there exist some $\tilde{\chi}_{j} \in C_{0}^{\infty}(B)$ equal to 1 near the support of $\chi_{j}$ such that

$$
\mathcal{E}_{h}^{j, 1} \leq C h^{-d} \int_{B} \tilde{\chi}_{j}(x)\left|u_{j}(x)\right|^{2}\left(\int_{B} 1_{|x-y| \leq h}|x-y|^{2} \mid d y\right) d x \leq C h^{2}\|u\|_{L^{2}(\Omega)}^{2}
$$

In order to estimate $\mathcal{E}_{h}^{j, 2}$ one has to estimate the contribution of the points $x \in \Omega, y \notin \Omega$ and $x \notin \Omega, y \notin \Omega$. All the terms are treated in the same way and we only examine

$$
\begin{aligned}
\mathcal{E}_{h}^{j, 3} & =h^{-d} \int_{\Omega \times(B \backslash \Omega)} 1_{|x-y| \leq h}\left|\chi_{j}(y)\right|^{2}\left|u_{j}(x)-u_{j}(y)\right|^{2} d x d y \\
& =h^{-d} \int_{\tilde{\Omega}_{j}^{+} \times \Omega_{j}^{-}} 1_{|x-y| \leq h}\left|\chi_{j}(y)\right|^{2}\left|u(x)-u \circ \phi_{j} \circ S \circ \phi_{j}^{-1}(y)\right|^{2} d x d y
\end{aligned}
$$

Let $\sigma: \mathbb{R}^{d} \rightarrow \mathbb{R}^{d}$ be the symetry with respect to $\left\{y_{d}=0\right\}$, so that $S \sigma=I d$ on $\left\{y_{d}<0\right\}$. We use the Lipschitz-continuous change of variable $\psi_{j}: y \in \Omega_{j}^{+} \mapsto \phi_{j} \circ \sigma \circ \phi_{j}^{-1}(y) \in \Omega_{j}^{-}$ to get

$$
\mathcal{E}_{h}^{j, 3} \leq C h^{-d} \int_{\tilde{\Omega}_{j}^{+} \times \Omega_{j}^{+}} 1_{\left|x-\psi_{j}(y)\right| \leq h}\left|\chi_{j} \circ \psi_{j}(y)\right|^{2}|u(x)-u(y)|^{2} d x d y
$$

We claim that there exists $\beta>0$ such that,

$$
\forall(x, y) \in \tilde{\Omega}_{j}^{+} \times \Omega_{j}^{+},\left|\psi_{j}(y)-x\right| \geq \beta^{-1}|x-y| .
$$

Inded, as both $\phi_{j}$ and $\phi_{j}^{-1}$ are Lipschitz-continuous, $\frac{2.8}{2.26}$ is equivalent to find $\beta>0$ such that

$$
\forall(x, y) \in \tilde{\Omega}_{j}^{+} \times \Omega_{j}^{+},|\sigma(x)-y| \geq \beta^{-1}|x-y|
$$


wich is obvious with $\beta=1$. From $\left(\frac{2.8}{2.26}\right)$ it follows that for some $\alpha>1$, one has

$$
\mathcal{E}_{h}^{j, 3} \leq C h^{-d} \int_{\tilde{\Omega}_{j}^{+} \times \Omega_{j}^{+}} 1_{|x-y| \leq \alpha h}|u(z)-u(y)|^{2} d z d y \leq C \mathcal{E}_{\alpha h, \rho}(u)
$$

and the upper bound is then a straightforward consequence of Lemma $\frac{1 \mathrm{em0}}{2.1 .}$

The byproduct is obtain by projecting the extension $v=E(u)$ on low frequencies $h|k| \leq 1$ and high frequencies $h|k|>1$ and the fact that the function $\theta$ is quadratic near 0 and has a positive lower bound for $|\xi| \geq 1$. The proof of lemma $\frac{1.2}{2.2}$ is complete.

We are in position to prove the estimate $\left(\frac{\text { gap3 } 3}{1.6)^{3}}\right.$ on the spectral gap. To show the right inequality, it suffices to plug a function $u \in C_{0}^{\infty}(\Omega)$ into (1.3.3) with support contained in a small ball $Q \subset \Omega$ and such that $\int_{\Omega} u(x) \rho(x) d x=0$. As $Q$ is convex, it follows from Taylor formula that for such $u$, we have $\left\langle u-T_{h} u, u\right\rangle=O\left(h^{2}\right)$.

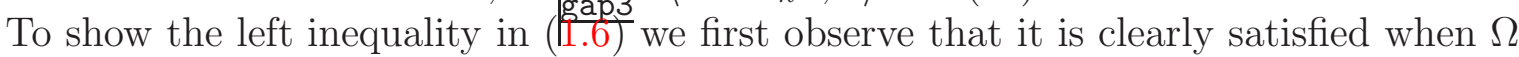
is convex. Indeed, given $u \in L^{2}(\Omega)$ we have

$$
\|u\|_{L^{2}(\Omega)}^{2}-\langle u, 1\rangle^{2} \leq C h^{-1} \sum_{k=0}^{K(h)} \int_{\Omega \times \Omega}(u(x+k h(y-x) / \alpha)-u(x+(k+1) h(y-x) / \alpha))^{2} d x d y
$$

where $\alpha=\operatorname{diam}(\Omega)$ and $K(h)=O\left(h^{-1}\right)$. With the new variables $x^{\prime}=x+k h(y-x) / \alpha$, $y^{\prime}=x+(k+1) h(y-x) / \alpha$ it comes

$$
\|u\|_{L^{2}(\Omega)}^{2}-\langle u, 1\rangle^{2} \leq C \alpha h^{-d-1} K(h) \int_{\Omega \times \Omega} 1_{\left|x^{\prime}-y^{\prime}\right|<h}\left(u\left(x^{\prime}\right)-u\left(y^{\prime}\right)\right)^{2} d x^{\prime} d y^{\prime}
$$

which proves the left inequality in $\left(\frac{\text { gap } 3}{1.6)}\right.$ in the case where $\Omega$ is convex.

In the general case, we can find some open sets contained in $\Omega, \omega_{j} \subset \subset \Omega_{j}^{+} \subset \subset \tilde{\Omega}_{j}^{+}$, $j=1, \ldots, N+M$ such that for $j=1, \ldots, N, \Omega_{j}^{+}, \tilde{\Omega}_{j}^{+}$are given in the previous lemma, $\left(\Omega_{j}^{+}\right)_{j=N+1, \ldots, N+M}$ are convex $\Omega_{0} \subset \cup_{j=N+1}^{M} \Omega_{j}^{+}, \Omega \subset \cup_{j=1}^{N+M} \omega_{j}$, and where $A \subset \subset B$ that $\bar{A}^{\Omega} \subset B$. Hence for $h>0$ small enough

$$
\begin{aligned}
\mathcal{E}_{h, \rho}(u) \geq C \sum_{j=1}^{N+M} h^{-d} \int_{\Omega_{j}^{+} \times \tilde{\Omega}_{j}^{+}} 1_{|x-y|<h}(u(x)-u(y))^{2} d x d y \\
\geq C \sum_{j=1}^{N} h^{-d} \int_{Q_{j} \times \tilde{Q}_{j}} 1_{\left|\phi_{j}(x)-\phi_{j}(y)\right|<h}\left(u \circ \phi_{j}(x)-u \circ \phi_{j}(y)\right)^{2} d x d y \\
\quad+C \sum_{j=N+1}^{N+M} h^{-d} \int_{\Omega_{j}^{+} \times \tilde{\Omega}_{j}^{+}} 1_{|x-y|<h}(u(x)-u(y))^{2} d x d y
\end{aligned}
$$

From the estimate proved precedently in the convex case, we know that there exists $a>0$ independant on $h$ such that the second sum in $\left(\frac{e q 2.9}{2.31}\right)$ is bounded from below by

$$
C h^{2} \sum_{j=N+1}^{N+M} \int_{\omega_{j} \times \Omega_{j}^{+}}(u(x)-u(y))^{2} d x d y \geq C h^{2} \sum_{j=N+1}^{N+M} \int_{\omega_{j} \times \Omega,|x-y|<a}(u(x)-u(y))^{2} d x d y .
$$


On the other hand, thanks to the fact that $\phi_{j}$ is Lipschitz diffeomorphism, there exists $\alpha>0$ such that $1_{x-y \mid<h / \alpha} \leq 1_{\left|\phi_{j}(x)-\phi_{j}(y)\right|<h} \leq 1_{|x-y|<\alpha h}$. Using the convexity of $Q_{i}$ and Lemma $\frac{1 e m 0}{2.1}$ it follows that the first sum in the right hand side of $\left(\frac{2 q 2.3)}{2.31}\right.$ is bounded from below by

$$
C h^{2} \sum_{j=1}^{N} \int_{\omega_{j} \times \Omega,|x-y|<a}(u(x)-u(y))^{2} d x d y
$$

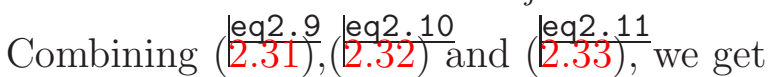

$$
\mathcal{E}_{h, \rho}(u) \geq C h^{2} \int_{\Omega \times \Omega,|x-y|<a}(u(x)-u(y))^{2} d x d y
$$

for some fixed $a>0$ independant on $h$. Using Lemma $\frac{\mid \text { lem0 }}{2.1}$ with $h=a$ we achieve the proof of (li.b).

lem3 Lemma 2.3 There exists $\left.\delta_{0} \in\right] 0,1 / 2\left[\right.$ such that $\operatorname{Spec}\left(T_{h, \rho}\right) \cap\left[1-\delta_{0}, 1\right]$ is discrete, and for any $0 \leq \lambda \leq \delta_{0} / h^{2}$, the number of eigenvalues of $T_{h}$ in $\left[1-h^{2} \lambda, 1\right]$ (with multiplicity) is bounded by $C_{1}(1+\lambda)^{d / 2}$. Moreover, any eigenfuntion $T_{h}(u)=\lambda u$ with $\lambda \in\left[1-\delta_{0}, 1\right]$ satisfies the bound

$$
\|u\|_{L^{\infty}} \leq C_{2} h^{-d / 2}\|u\|_{L^{2}}
$$

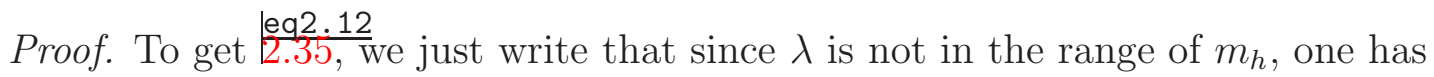

$$
u(x)=\frac{1}{\lambda-m_{h}(x)} \int_{\Omega} h^{-d} \varphi\left(\frac{x-y}{h}\right) \min \left(\frac{\rho(y)}{\rho(x)}, 1\right) u(y) d y
$$

and we apply Cauchy-Schwarz. The important point here is the estimate on the number of eigenvalues in $\left[1-h^{2} \lambda, 1\right]$ by a power of $\lambda$. This is obtain by the min-max and uses 2.20. The min-max gives: if for some closed subspace $F$ of $L^{2}(\rho)$ with $\operatorname{codim}(F)=N$ one has for all $u \in F, h^{-2}\left(\left(1-T_{h}\right) u \mid u\right)_{L^{2}(\rho)} \geq \lambda\|u\|_{L^{2}(\rho)}^{2}$, then the number of eigenvalues of $T_{h}$ in $\left[1-h^{2} \lambda, 1\right]$ (with multiplicity) is bounded by $\operatorname{codim}(F)=N$. Then, we fix $c>0$ small enough, and we choose for $F$ the subspace of functions $u$ such that their extension $v=E(u)$ is such that the Fourier coefficients satisfy $c_{k}(E(u))=0$ for $|k| \leq D$ with $h D \leq c$. The codimension of this space $F$ is exactly the number of $k \in \mathbb{Z}^{d}$ such that $|k| \leq D$, since if $p$ is a trigonometric polynomial such that $E^{*}(p)=0$, we will have $\int_{\Omega} p(x) u(x) d x=0$ for any function $u$ with compact support in $\Omega$ and such that $E(u)=u$, and this implies $p=0$. Thus $\operatorname{codim}(F) \simeq(1+D)^{d}$. On the other hand, the right inequality in 2.20 gives for $u \in F, h^{-2}\left(\left(1-T_{h}\right) u \mid u\right)_{L^{2}(\rho)} \geq C_{0}\left(D^{2}-C_{1}\right)\|u\|_{L^{2}(\rho)}^{2}$ for universal $C_{0}, C_{1}$, since by $\frac{2.6}{2.12}$, there exists $C_{0}>0$ such that one has $\theta(h k) h^{-2} \geq C_{0} D^{2}$ for all $D \leq c / h$ and all $|k|>D$. The proof of our lemma is complete.

We are now ready to prove the total variation estimate $\frac{1.7}{1.7}$. Let $\Pi_{0}$ be the orthogonal projector in $L^{2}(f)$ on the space of constant functions

$$
\Pi_{0}(u)(x)=1_{\Omega}(x) \int_{\Omega} u(y) \rho(y) d y
$$

Then

$$
2 \sup _{x_{0} \in \Omega}\left\|T_{h, x_{0}}^{n}-\rho(x) d x\right\|_{T V}=\left\|T_{h}^{n}-\Pi_{0}\right\|_{L^{\infty} \rightarrow L^{\infty}}
$$


Thus, we have to prove that there exist $C_{0}, h_{0}$, such that for any $n$ and any $\left.\left.h \in\right] 0, h_{0}\right]$, one has

$$
\left\|T_{h}^{n}-\Pi_{0}\right\|_{L^{\infty} \rightarrow L^{\infty}} \leq C_{0} e^{-n g_{h, \rho}}
$$

Observe that since we know that for $h_{0}$ small, the estimate 1.6 hap 3 holds true for any $\rho$, we may assume $n \geq C h^{-2}$. In order to prove 2.38 , we split $T_{h}$ in 3 pieces, according to the spectral theory.

Let $0<\lambda_{1, h} \leq \ldots \leq \lambda_{j, h} \leq \lambda_{j+1, h} \leq \ldots \leq h^{-2} \delta_{0}$ be such that the eigenvalues of $T_{h}$ in the interval $\left[1-\delta_{0}, 1\left[\right.\right.$ are the $1-h^{2} \lambda_{j, h}$, with associated orthonormals eigenfunctions $e_{j, h}$

$$
T_{h}\left(e_{j, h}\right)=\left(1-h^{2} \lambda_{j, h}\right) e_{j, h}, \quad\left(e_{j, h} \mid e_{k, h}\right)_{L^{2}(\rho)}=\delta_{j, k}
$$

Then we write $T_{h}-\Pi_{0}=T_{h, 1}+T_{h, 2}+T_{h, 3}$ with

$$
\begin{aligned}
& T_{h, 1}(x, y)=\sum_{\lambda_{1, h} \leq \lambda_{j, h} \leq h^{-\alpha}}\left(1-h^{2} \lambda_{j, h}\right) e_{j, h}(x) e_{j, h}(y) \\
& T_{h, 2}(x, y)=\sum_{h^{-\alpha}<\lambda_{j, h} \leq h^{-2} \delta_{0}}\left(1-h^{2} \lambda_{j, h}\right) e_{j, h}(x) e_{j, h}(y) \\
& T_{h, 3}=T_{h}-\Pi_{0}-T_{h, 1}-T_{h, 2}
\end{aligned}
$$

Here $\alpha>0$ is a small constant that will be chosen later. One has $T_{h}^{n}-\Pi_{0}=T_{h, 1}^{n}+T_{h, 2}^{n}+T_{h, 3}^{n}$, and we will get the bound 2.38 for each of the 3 terms. We start by very rough bounds. Since there is at most $C h^{-d}$ eigenvalues $\lambda_{j, h}$ and using the bound $\left(\frac{\mathrm{eq} 2.12}{2.35}\right)$, we get that there exists $C$ independent of $n \geq 1$ and $h$ such that

$$
\left\|T_{1, h}^{n}\right\|_{L^{\infty} \rightarrow L^{\infty}}+\left\|T_{2, h}^{n}\right\|_{L^{\infty} \rightarrow L^{\infty}} \leq C h^{-3 d / 2}
$$

Since $T_{h}^{n}$ is bounded by 1 on $L^{\infty}$, we get from $T_{h}^{n}-\Pi_{0}=T_{h, 1}^{n}+T_{h, 2}^{n}+T_{h, 3}^{n}$

$$
\left\|T_{3, h}^{n}\right\|_{L^{\infty} \rightarrow L^{\infty}} \leq C h^{-3 d / 2}
$$

Next we use $\frac{1}{1.2}$.2 to write $T_{h}=m_{h}+R_{h}$ with

$$
\begin{aligned}
& \left\|m_{h}\right\|_{L^{\infty} \rightarrow L^{\infty}} \leq \gamma<1 \\
& \left\|R_{h}\right\|_{L^{2} \rightarrow L^{\infty}} \leq C_{0} h^{-d / 2}
\end{aligned}
$$

From this, we deduce that for any $p=1,2, \ldots$, one has $T_{h}^{p}=A_{p, h}+B_{p, h}$, with $A_{1, h}=$ $m_{h}, B_{1, h}=R_{h}$ and the recurrence relation $A_{p+1, h}=m_{h} A_{p, h}, B_{p+1, h}=m_{h} B_{p, h}+R_{h} T_{h}^{p}$. Thus one gets since $T_{h}^{p}$ is bounded by 1 on $L^{2}$

$$
\begin{aligned}
& \left\|A_{p, h}\right\|_{L^{\infty} \rightarrow L^{\infty}} \leq \gamma^{p} \\
& \left\|B_{p, h}\right\|_{L^{2} \rightarrow L^{\infty}} \leq C_{0} h^{-d / 2}\left(1+\gamma+\ldots+\gamma^{p}\right) \leq C_{0} h^{-d / 2} /(1-\gamma)
\end{aligned}
$$

Let $\theta=1-\delta_{0}<1$ so that $\left\|T_{3, h}\right\|_{L^{2} \rightarrow L^{2}} \leq \theta$. Then one has

$$
\left\|T_{3, h}^{n}\right\|_{L^{\infty} \rightarrow L^{2}} \leq\left\|T_{3, h}^{n}\right\|_{L^{2} \rightarrow L^{2}} \leq \theta^{n}
$$

and for $n \geq 1, p \geq 1$, one gets using $\frac{T 9}{2.44}$ and $\frac{T 7}{2.42}$ 


$$
\begin{aligned}
& \left\|T_{3, h}^{p+n}\right\|_{L^{\infty} \rightarrow L^{\infty}}=\left\|T_{h}^{p} T_{3, h}^{n}\right\|_{L^{\infty} \rightarrow L^{\infty}} \\
& \leq\left\|A_{p, h} T_{3, h}^{n}\right\|_{L^{\infty} \rightarrow L^{\infty}}+\left\|B_{p, h} T_{3, h}^{n}\right\|_{L^{\infty} \rightarrow L^{\infty}} \\
& \leq C h^{-3 d / 2} \gamma^{p}+C_{0} h^{-d / 2} \theta^{n} /(1-\gamma)
\end{aligned}
$$

Thus we get for some $C>0, \mu>0$,

$$
\left\|T_{3, h}^{n}\right\|_{L^{\infty} \rightarrow L^{\infty}} \leq C e^{-\mu n}, \quad \forall h, \quad \forall n \geq 1 / h
$$

and thus the contribution of $T_{3, h}^{n}$ is far smaller than the bound we have to prove in $\frac{T 3}{2.38}$. Next, for the contribution of $T_{\text {. }}^{n}$, we just write, since there is at most $C h^{-d}$ eigenvalues $\lambda_{j, h}$ and using the bound $\left(\frac{e q 2.35)}{2.35}\right.$

$$
\begin{aligned}
& T_{h, 2}^{n}(x, y)=\sum_{h^{-\alpha}<\lambda_{j, h} \leq h^{-2} \delta_{0}}\left(1-h^{2} \lambda_{j, h}\right)^{n} e_{j, h}(x) e_{j, h}(y) \\
& \left\|T_{2, h}^{n}\right\|_{L^{\infty} \rightarrow L^{\infty}} \leq C h^{-3 d / 2}\left(1-h^{2-\alpha}\right)^{n}
\end{aligned}
$$

Thus we get for some $C_{\alpha}>0$,

$$
\left\|T_{2, h}^{n}\right\|_{L^{\infty} \rightarrow L^{\infty}} \leq C_{\alpha} e^{-\frac{n h^{2-\alpha}}{2}}, \quad \forall h, \quad \forall n \geq h^{-2+\alpha / 2}
$$

and thus this contribution is still neglectible for $\left.h \in] 0, h_{0}\right]$ for $h_{0}$ small. It remains to study the contribution of $T_{h, 1}^{n}$. Let $E_{\alpha}$ be the (finite dimensional) subspace of $L^{2}(\rho)$ span by the eigenvectors $e_{j, h}, \lambda_{j, h} \leq h^{-\alpha}$. By lemma $\frac{1 \mathrm{lem} 3}{2.3,}$ one has $\operatorname{dim}\left(E_{\alpha}\right) \leq C h^{-d \alpha / 2}$.

lem4 Lemma 2.4 There exist $\alpha>0, p>2$ and $C$ independent of $h$ such that for all $u \in E_{\alpha}$, the following inequality holds true

$$
\|u\|_{L^{p}}^{2} \leq C h^{-2}\left(\left(\mathcal{E}_{\Omega, h}(u)+h^{2}\|u\|_{L^{2}}^{2}\right)\right.
$$

Proof. Clearly, one has for $u=\sum_{\lambda_{1, h} \leq \lambda_{j, h} \leq h^{-\alpha}} a_{j} e_{j, h} \in E_{\alpha}$

$$
\mathcal{E}_{\Omega, h}(u)+h^{2}\|u\|_{L^{2}}^{2}=\sum_{\lambda_{1, h} \leq \lambda_{j, h} \leq h^{-\alpha}} h^{2}\left(1+\lambda_{j, h}\right)\left|a_{j}\right|^{2}
$$

Take $u \in E_{\alpha}$ such that $h^{-2}\left(\left(\mathcal{E}_{\Omega, h}(u)+h^{2}\|u\|_{L^{2}}^{2}\right) \leq 1\right.$. Then by 2.720 , one has $h^{-2} \mathcal{E}_{h}(E(u)) \leq$ $C_{0}$. Let $\psi(t) \in C_{0}^{\infty}(\mathbb{R})$ equal to 1 near $t=0$, and for $v(x)=\sum_{k \in \mathbb{Z}^{d}} c_{k}(v) e^{2 i \pi k x / A}$, set

$$
v=v_{L}+v_{H}, \quad v_{L}(x)=\sum_{k \in \mathbb{Z}^{d}} \psi(h|k|) c_{k}(v) e^{2 i \pi k x / A}
$$

Then $v=v_{L}+v_{H}$ is a decomposition of the extension $v=E(u)$ in low frequencies $\left(v_{L}\right)$ and high frequencies $\left(v_{H}\right)$. One has $v_{L}(x)=\int_{\mathbb{R}^{d}} h^{-d} \theta\left(\frac{x-y}{h}\right) v(y) d y$, where $\theta$ is the function in the Schwartz space defined by $\hat{\theta}(2 \pi z / A)=\psi(|z|)$. Hence, the map $v \mapsto v_{L}$ is bounded uniformly in $h$ on all the space $L^{q}$ for $1 \leq q \leq \infty$. Then, from $\frac{2.6}{2.12}$ we get

$$
\left\|v_{L}\right\|_{H^{1}(B)} \leq C
$$

Thus, with $u_{L}=\left.v_{L}\right|_{\Omega}$ and $u_{H}=\left.v_{H}\right|_{\Omega}$, we get $\left\|u_{L}\right\|_{H^{1}(\Omega)} \leq C$ so by Sobolev for $p<\frac{2 d}{d-2}$

$$
\left\|u_{L}\right\|_{L^{p}} \leq C
$$


One the other hand, one has also by $\frac{2.7}{2.20}$

$$
h^{-2} \mathcal{E}_{h}\left(E\left(e_{j, h}\right)\right) \leq C_{0}\left(1+\lambda_{j, h}\right)
$$

and this implies by $\frac{2.6}{2.12}$

$$
h^{-2}\left\|E\left(e_{j, h}\right)_{H}\right\|_{L^{2}}^{2} \leq C_{0}\left(1+\lambda_{j, h}\right) \leq C_{0}\left(1+h^{-\alpha}\right)
$$

Thus for $\alpha \leq 1$, we get $\left\|E\left(e_{j, h}\right)_{H}\right\|_{L^{2}} \leq C h^{1 / 2}$. On the other hand, since $\left\|e_{j, h}\right\|_{L^{\infty}} \leq$ $C h^{-d / 2}$, using the definition of the low frequency cut-off we get ,

$$
\left\|E\left(e_{j, h}\right)_{H}\right\|_{L^{\infty}} \leq\left\|E\left(e_{j, h}\right)\right\|_{L^{\infty}}+\left\|E\left(e_{j, h}\right)_{L}\right\|_{L^{\infty}} \leq C\left\|E\left(e_{j, h}\right)\right\|_{L^{\infty}} \leq C h^{-d / 2}
$$

By interpolation we can find some $p>2$ such that

$$
\left\|E\left(e_{j, h}\right)_{H}\right\|_{L^{p}} \leq C_{0} h^{1 / 4}
$$

Thus, one get for $u=\sum_{\lambda_{1, h} \leq \lambda_{j, h} \leq h^{-\alpha}} a_{j} e_{j, h} \in E_{\alpha}$ with $h^{-2}\left(\left(\mathcal{E}_{\Omega, h}(u)+h^{2}\|u\|_{L^{2}}^{2}\right) \leq 1\right.$

$$
\begin{aligned}
& \left\|u_{H}\right\|_{L^{p}} \leq \sum_{\lambda_{1, h} \leq \lambda_{j, h} \leq h^{-\alpha}}\left|a_{j}\right|\left\|E\left(e_{j, h}\right)_{H}\right\|_{L^{p}} \\
& \leq C_{0} h^{1 / 4} \operatorname{dim}\left(E_{\alpha}\right)^{1 / 2}\|u\|_{L^{2}} \leq C h^{1 / 4} h^{-d \alpha / 4}
\end{aligned}
$$

Our lemma follows from $\frac{T 16}{2.52}$ and $\frac{T 20}{2.56}$ if one takes $\alpha$ small. Observe that here, the estimate on the number of eigenvalues (i.e the estimation of the dimension of $E_{\alpha}$ ) is crucial. The proof of lemma $\frac{1 \mathrm{em} 4}{2.4 \mathrm{is}}$ complete.

From lemma $\frac{1 \mathrm{em} 4}{2.4,}$, using the interpolation inequality $\|u\|_{L^{2}}^{2} \leq\|u\|_{L^{p}}^{\frac{p}{p-1}}\|u\|_{L^{1}}^{\frac{p-2}{p-1}}$, we deduce the Nash inequality, with $1 / D=2-4 / p>0$

$$
\|u\|_{L^{2}}^{2+1 / D} \leq C h^{-2}\left(\left(\mathcal{E}_{\Omega, h}(u)+h^{2}\|u\|_{L^{2}}^{2}\right)\|u\|_{L^{1}}^{1 / D}, \quad \forall u \in E_{\alpha}\right.
$$

For $\lambda_{j, h} \leq h^{-\alpha}$, one has $h^{2} \lambda_{j, h} \underset{\text { T20bis }}{1} 1_{\text {and }}$ and thus for any $u \in E_{\alpha}$, one gets $\mathcal{E}_{\Omega, h}(u) \leq$

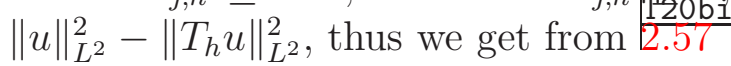

$$
\|u\|_{L^{2}}^{2+1 / D} \leq C h^{-2}\left(\left(\|u\|_{L^{2}}^{2}-\left\|T_{h} u\right\|_{L^{2}}^{2}+h^{2}\|u\|_{L^{2}}^{2}\right)\|u\|_{L^{1}}^{1 / D}, \quad \forall u \in E_{\alpha}\right.
$$

From $\frac{T 11}{2.46}$ and $\frac{T 13}{2.48}$, and $T_{h}^{n}-\Pi_{0}=T_{h, 1}^{n}+T_{h, 2}^{n}+T_{h, 3}^{n}$, we get that there exists $C_{2}$ such that

$$
\left\|T_{1, h}^{n}\right\|_{L^{\infty} \rightarrow L^{\infty}} \leq C_{2}, \quad \forall h, \quad \forall n \geq h^{-2+\alpha / 2}
$$

and thus since $T_{1, h}$ is self adjoint on $L^{2}$

$$
\left\|T_{1, h}^{n}\right\|_{L^{1} \rightarrow L^{1}} \leq C_{2}, \quad \forall h, \quad \forall n \geq h^{-2+\alpha / 2}
$$

Fix $p \simeq h^{-2+\alpha / 2}$. Take $g \in L^{2}$ such that $\|g\|_{L^{1}} \leq 1$ and consider the sequence $c_{n}, n \geq 0$

$$
c_{n}=\left\|T_{1, h}^{n+p} g\right\|_{L^{2}}^{2}
$$

Then, $0 \leq c_{n+1} \leq c_{n}$ and from $\frac{T 21}{2.58}$ and $\frac{T 23}{2.60}$, we get

$$
\begin{aligned}
& c_{n}^{1+\frac{1}{2 D}} \leq C h^{-2}\left(c_{n}-c_{n+1}+h^{2} c_{n}\right)\left\|T_{1, h}^{n+p} g\right\|_{L^{1}}^{1 / D} \\
& \leq C C_{2}^{1 / D} h^{-2}\left(c_{n}-c_{n+1}+h^{2} c_{n}\right)
\end{aligned}
$$


From this inequality, we deduce that there exist $A \simeq C C_{2} \sup _{0 \leq n \leq h^{-2}}(2+n)\left(1+h^{2}-\right.$ $\left(1-\frac{1}{n+2}\right)^{2 D}$ ) which depends only on $C, C_{2}, D$, such that for all $0 \leq n \leq h^{-2}$, one has $c_{n} \leq\left(\frac{A h^{-2}}{1+n}\right)^{2 D}$, and thus there exist $C_{0}$ which depends only on $C, C_{2}, D$, such that for $N \simeq h^{-2}$, one has $c_{N} \leq C_{0}$. This implies

$$
\left\|T_{1, h}^{N+p} g\right\|_{L^{2}} \leq C_{0}\|g\|_{L^{1}}
$$

and thus taking adjoints

$$
\left\|T_{1, h}^{N+p} g\right\|_{L^{\infty}} \leq C_{0}\|g\|_{L^{2}}
$$

and so we get for any $n$ and with $N+p \simeq h^{-2}$

$$
\left\|T_{1, h}^{N+p+n} g\right\|_{L^{\infty}} \leq C_{0}\left(1-h^{2} \lambda_{1, h}\right)^{n}\|g\|_{L^{2}}
$$

And thus for $n \geq h^{-2}$

$$
\left\|T_{1, h}^{n}\right\|_{L^{\infty} \rightarrow L^{\infty}} \leq C_{0} e^{-\left(n-h^{-2}\right) h^{2} \lambda_{1, h}}=C_{0} e^{\lambda_{1, h}} e^{-n g a p}, \quad \forall h, \quad \forall n \geq h^{-2}
$$

This conclude the proof of theorem $\frac{\operatorname{thm} 1}{1.1 .}$

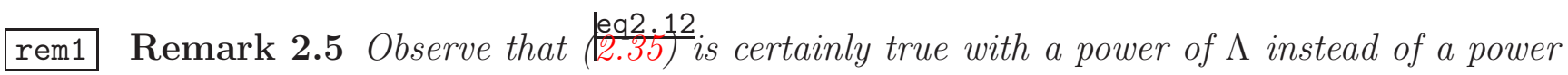
of $h$ with $\lambda=1-h^{2} \Lambda$, but we have no proof for this; thats why we use for $T_{1, h}$ a Nash inequality.

rem2 Remark 2.6 The above proof seems to apply for a more general choice of the elementary Markov kernel $h^{-d} \varphi\left(\frac{x-y}{h}\right)$. Replace $\varphi$ by a positive symmetric measure of total mass 1 with support in the unit ball, and let $T_{h}$ be the Metropolis with this data. Assume that one is able to prove that for some $\delta_{0}>0$ one has $\operatorname{Spec}\left(T_{h}\right) \subset\left[-1+\delta_{0}, 1\right]$ for all $h \leq h_{0}$, and that for some power $M$, one has for some $C, c>0$

$$
T_{h}^{M}(x, d y)=\mu_{h}(x, d y)+C h^{-d} 1_{|x-y| \leq c h} \rho(y) d y, \quad \mu_{h}(x, d y) \geq 0
$$

Then there exist $\gamma<1$ such that $\left\|\mu_{h}\right\|_{L^{\infty}} \leq \gamma$. Moreover, the right inequality in $\frac{2.7}{2.20}$ and $\frac{\mathrm{eq} 2.12}{2.35}$ are still valid for $T_{h}^{M}$. Also, the spectral gap of $T_{h}^{M}$ is given by formula 1.4 with $T_{h}^{M}(x, d y)$ in place of $K_{h, \rho}(x, y) d y$, and therefore the left inequality in 1.6 holds true, and the right one is true, since if $\rho$ is constant, for any $\theta \in C_{0}^{\infty}(\Omega)$, one has $u-T_{h} u \in O_{c}\left(h^{2}\right)$. We shall use these remark later in the study of the hard disc problem, in section $\frac{\text { sect. }}{4 .}$

\section{A proof of theorem $\frac{\operatorname{thm} 2}{1.2}$}

In all this section we suppose additionaly that $\Omega$ is stratified. For a given continuous density $\rho$, using (2.4) and an approximation of $\rho$ in $L_{\operatorname{thm} 2}^{\infty}$ by a sequence of smooth density $\rho_{k}$, one ssees that the first assertion (1. (18) of theorem $\frac{1.6}{1.2}$ is a consequence of the second one (1.7). Assume now that $\rho$ is smooth.

lem5 Lemma 3.1 Let $\theta \in C^{\infty}(\bar{\Omega})$ be such that $\operatorname{supp}(\theta) \cap \Gamma_{\text {sing }}=\emptyset$ and $\left.\partial_{n} \theta\right|_{\Gamma_{\text {reg }}}=0$. Then

$$
Q_{h, \rho}(\theta)=h^{2} L_{\rho}(\theta)+r, \quad\|r\|_{L^{2}} \in O\left(h^{5 / 2}\right)
$$


Proof. For $\theta \in C^{\infty}(\bar{\Omega})$ and $x \in \Omega$, we can use the Taylor formula to get

$$
\begin{array}{r}
Q_{h, \rho}(\theta)(x)=\frac{1}{V o l\left(B_{1}\right)} \int_{A(x, h)} \min \left(1+h \frac{\nabla \rho(x)}{\rho(x)} \cdot z+O\left(h^{2}|z|^{2}\right), 1\right) \\
\left(-h \nabla \theta(x) \cdot z-\frac{h^{2}}{2} \sum_{i, j} z_{i} z_{j} \partial_{x_{i}} \partial_{x_{j}} \theta(x)+O\left(h^{3}|z|^{3}\right)\right) d z
\end{array}
$$

with $A(x, h)=\left\{z \in \mathbb{R}^{d},|z|<1, x+h z \in \Omega\right\}$. As $A(x, h)=A^{+}(x, h) \cup A^{-}(x, h)$, with $A^{ \pm}(x, h)=\{z \in A(x, h), \pm(\rho(x+h z)-\rho(x)) \geq 0\}$, it follows by an easy computation that

$$
\begin{aligned}
Q_{h, \rho}(\theta)(x)= & -\frac{h}{\operatorname{Vol}\left(B_{1}\right)} \nabla \theta(x) \cdot \int_{A(x, h)} z d z-\frac{h^{2}}{2 \operatorname{Vol}\left(B_{1}\right)} \sum_{i, j=1}^{d} \partial_{x_{i}} \partial_{x_{j}} \theta(x) \int_{A(x, h)} z_{i} z_{j} d z \\
& -\frac{h^{2}}{\operatorname{Vol}\left(B_{1}\right)} \int_{A^{-}(x, h)} \frac{\nabla \rho(x)}{\rho(x)} \cdot z \nabla \theta(x) . z d z+r(x)=f_{1}(x)+f_{2}(x)+f_{3}(x)+r(x)
\end{aligned}
$$

with $\|r\|_{L^{\infty}(\Omega)}=O\left(h^{3}\right)$. Let $\chi=1_{d(x, \partial \Omega)<2 h}$, then for $j=2,3$

$$
\left\|\chi f_{j}\right\|_{L^{2}(\Omega)} \leq\|\chi\|_{L^{2}(\Omega)}\left\|f_{j}\right\|_{L^{\infty}}(\Omega)=O\left(h^{5 / 2}\right)
$$

thanks to the support properties of $\chi$. Moreover, for $x \in \operatorname{supp}(1-\chi), A(x, h)=\{|z|<1\}$ and the change of variable $z \mapsto-z$ shows that $(1-\chi) f_{2}=-(1-\chi) \frac{\alpha_{d}}{2} h^{2} \Delta \theta(x)$ thanks to 1..8. Hence,

$$
f_{2}(x)=-\frac{\alpha_{d}}{2} h^{2} \Delta \theta(x)+r(x)
$$

with $\|r\|_{L^{2}}=O\left(h^{5 / 2}\right)$.

To compute $f_{3}$, we first observe that $\left|f_{3}(x)\right| \leq C h^{2}|\nabla \rho(x)||\nabla \theta(x)|$. We thus get $\left\|1_{|\nabla \rho| \leq h^{1 / 2}} f_{3}\right\|_{L^{\infty}} \leq C h^{5 / 2}\|\nabla \theta\|_{L^{\infty}}$. At a point $x$ where $|\nabla \rho(x)| \geq h^{1 / 2}$, we may write $z=t \frac{\nabla \rho(x)}{|\nabla \rho(x)|}+z^{\perp}, \quad t=\frac{z \cdot \nabla \rho(x)}{|\nabla \rho(x)|}, \quad z^{\perp} . \nabla \rho(x)=0$. In these coordinates, one has $A^{-}(x, h)=$ $\left\{\left(t, z^{\perp}\right), t|\nabla \rho(x)|+O\left(h\left(t^{2}+\left|z^{\perp}\right|^{2}\right)\right) \leq 0\right\}$. From $|\nabla \rho(x)| \geq h^{1 / 2}$ we get that the symmetric difference $R$ between $A^{-}(x, h)$ and $\{t \leq 0\}$ satisfies meas $(R)=O\left(h^{1 / 2}\right)$ (the symmetric difference of two sets $A, B$ is $A \cup B \backslash A \cap B)$. Therefore

$$
1_{|\nabla \rho| \geq h^{1 / 2}}(1-\chi) f_{3}(x)=-h^{2} 1_{|\nabla \rho| \geq h^{1 / 2}}(1-\chi)(x) \int_{\{|z|<1, \nabla \rho(x) \cdot z \leq 0\}} \frac{\nabla \rho(x)}{\rho(x)} \cdot z \nabla \theta(x) . z d z+r(x)
$$

with $\|r\|_{L^{\infty}}=O\left(h^{5 / 2}\right)$. Using the change of variable $z \mapsto z-2 z^{\perp}$ we get

$$
1_{|\nabla \rho| \geq h^{1 / 2}}(1-\chi) f_{3}(x)=-h^{2} 1_{|\nabla \rho| \geq h^{1 / 2}} \frac{\alpha_{d}}{2}(1-\chi)(x) \frac{\nabla \rho(x)}{\rho(x)} \cdot \nabla \theta(x)+r(x)
$$

and therefore using $\frac{3.1 \mathrm{bis}}{3.4 \mathrm{we}}$ get

$$
f_{3}(x)=-h^{2} \frac{\alpha_{d}}{2} \frac{\nabla \rho(x)}{\rho(x)} \cdot \nabla \theta(x)+r(x)
$$

with $\|r\|_{L^{2}}=O\left(h^{5 / 2}\right)$. It remains to show that $\left\|f_{1}\right\|_{L^{2}(\Omega)}=O\left(h^{5 / 2}\right)$. Using the change of variable $z \mapsto-z$ we easily obtain $(1-\chi) f_{1}=0$. Hence, it suffices to show that $f_{1}^{\prime}(x, h)=$ 
$\int_{A(x, h)} z \cdot \nabla \theta(x) d z$ satisfies $\left\|f_{1}^{\prime}\right\|_{L^{\infty}(\Omega)}=O(h)$. As $\Gamma_{\text {sing }}$ is compact and $\operatorname{supp}(\theta) \cap \Gamma_{\text {sing }}=\emptyset$, $\operatorname{dist}\left(\Gamma_{\text {sing }}, \operatorname{supp}(\theta)\right)>0$, this is a local problem near any point $x_{0}$ of the regular part $\Gamma_{\text {reg }}$

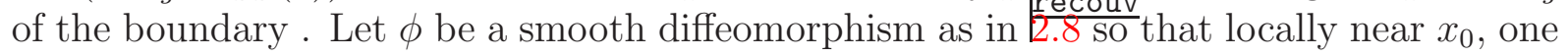
has $\phi^{-1}(\Omega)=\left\{x_{d}>0\right\}$. For $x$ close to $x_{0}$ one has

$$
A(x, h)=\left\{z \in \mathbb{R}^{d},|z|<1,\left(\phi^{-1}(x)+h D_{x} \phi^{-1}(z)+O\left(h^{2}\right)\right)_{d}>0\right\}
$$

Set

$$
A_{1}(x, h)=\left\{z \in \mathbb{R}^{d},|z|<1,\left(\phi^{-1}(x)+h D_{x} \phi^{-1}(z)\right)_{d}>0\right\}
$$

then the symmetric difference $R$ between $A(x, h)$ and $A_{1}(x, h)$ satisfies meas $(R)=O(h)$ uniformly in $x$ close to $x_{0}$. This yields

$$
f_{1}^{\prime}(x, h)=\nabla \theta(x) \cdot v(x, h)+r(x), \quad v(x, h)=\int_{A_{1}(x, h)} z d z
$$

with $\|r\|_{L^{\infty}}=O(h)$. Let $\nu(x)$ be the vector field defined by $\nu(x) . z=\left(D_{x} \phi^{-1}(x)(z)\right)_{d}$. Observe that $v(x, h)$ is collinear to $\nu(x)$, vanish for $\operatorname{dist}(x, \partial \Omega)>C h$ and that for $x \in \partial \Omega$, $\nu(x)$ is collinear to the unit normal to the boundary $\vec{n}(x)$. Since $\left.\partial_{n} \theta\right|_{\Gamma_{\text {reg }}}=0$, we thus get $\left\|f_{1}^{\prime}\right\|_{L^{\infty}}=O(h)$. The proof of our lemma is complete.

Let us recall that we denote $1=\nu_{0}<\nu_{1}<\ldots<\nu_{j}<\ldots$ the eigenvalues of $L_{\rho}$ and $m_{j}$ the associated multiplicities. We introduce the bilinear form

$$
a_{\rho}(u, v)=\frac{\alpha_{d}}{2}\langle\nabla u, \nabla v\rangle_{L^{2}(\rho)}+\langle u, v\rangle_{L^{2}(\rho)} .
$$

It defines an Hilbertian structure on $H^{1}(\Omega)$ which is equivalent to the usual one. We denote $\|\cdot\|_{H_{\rho}^{1}}$ the norm induced by $a_{\rho}$. For $j \in \mathbb{N}$ we denote $F_{j}=\operatorname{Ker}\left(L_{\rho}-\nu_{j}\right), F_{<N}=$ $\oplus_{j<N} F_{j}$ and by $F_{\geq N}=\oplus_{j \geq N} F_{j}$ the orthogonal complement of $F_{<N}$ in $H^{1}$ for teh scalarr product $a_{\rho}$. Observe that since we assume here $\rho$ smooth, by the classical theory of elliptic boundary problems, any function in $F_{j}$ is smooth in $\Omega$ and smooth up to the regular part $\Gamma_{r e g}$ of the boundary. We also denote $\Pi_{j}$ the orthogonal projection for $a_{\rho}$ on $F_{j}$ and

$$
\mathcal{D}_{N}=\left\{\theta \in C^{\infty}(\bar{\Omega}), \theta=0 \text { near } \Gamma_{\text {sing }}, \partial_{n} \theta_{\mid \Gamma_{\text {reg }}}=0,\langle\theta, v\rangle_{L^{2}(\rho)}=0 \quad \forall v \in F_{<N}\right\}
$$

where we use the convention $F_{\mathbb{1}^{0} 0_{3.4}} \emptyset$. One has $\mathcal{D}_{N} \subset F_{\geq N}$, since for any $\theta \in \mathcal{D}_{N}$ and any $v \in F_{j}$ with $j<N$ one has by $\frac{1.3 .14}{1.14} a_{\rho}(v, \theta)=\left\langle\left(L_{\rho}+1\right) v, \theta\right\rangle_{L^{2}(\rho)}=\left\langle\left(\nu_{j}+1\right) v, \theta\right\rangle_{L^{2}(\rho)}=0$.

lem6 Lemma 3.2 For all $N \in \mathbb{N}$ and all $u \in F_{\geq N}$ there exists a sequence $\left(u_{k}\right)$ in $\mathcal{D}_{N}$ converging to $u$ in $H^{1}$.

Proof. We proceed by induction. Let us first verify the property for $N=0$, i.e that $\mathcal{D}_{0}$ is dense in $H^{1}$. Let $f \in H^{1}(\Omega)$ be orthogonal to $\mathcal{D}_{0}$ for $a_{\rho}$. Then, it is orthogonal to $C_{0}^{\infty}(\Omega)$ so that $\left(L_{\rho}+1\right) f=0$ in the sense of distributions. In particular $-\Delta f \in L^{2}(\Omega)$. Hence we can use the Green formula (1.14) to get for any $\theta \in \mathcal{D}_{0}$, since $a_{\rho}(f, \theta)=0$,

$$
\left\langle\partial_{n} f, \rho \theta\right\rangle_{H^{-1 / 2}, H^{1 / 2}}=0
$$

For any $\psi \in C_{0}^{\infty}\left(\Gamma_{\text {reg }}\right)$, using smooth local coordinates we can find $\tilde{\psi}$ in $\mathcal{D}_{0}$ such that $\tilde{\psi}_{\mid \partial \Omega}=\psi$. Consequently,

$$
\left\langle\partial_{n} f, \rho \psi\right\rangle_{H^{-1 / 2}, H^{1 / 2}}=\left\langle\partial_{n} f, \rho \tilde{\psi}\right\rangle_{H^{-1 / 2}, H^{1 / 2}}=0
$$


Hence, $\partial_{n} f_{\mid \Gamma_{\text {reg }}}=0$. This shows that $\partial_{n} f_{\mid \partial \Omega} \in H^{-1 / 2}$ is supported in $\Gamma_{\text {sing. }}$. From $\frac{\text { unig }}{1.16}$ this implies $\partial_{n} f_{\mid \partial \Omega}=0$. This shows that $f \in D\left(L_{\rho}\right)$. As the operator $L_{\rho}+1$ is strictly positive this implies $f=0$.

For $N \geq 1$ and $f \in F_{\geq N}$, we consider a familly $\left(f_{\epsilon}\right)$ in $\mathcal{D}_{0}$ such that $\left\|f-f_{\epsilon}\right\|_{H^{1}} \leq \epsilon$. Let $Q$ be an open ball such that $\bar{Q} \subset \Omega$. We look for $h \in C_{0}^{\infty}(Q)$ such that $\tilde{f}_{\epsilon}=f_{\epsilon}+h$ satisfies the lemma. Let $\theta \in C_{0}^{\infty}(Q)$, with $\theta \geq 0$ and $\theta \neq 0$. We look for $h$ under the form $h=\sum_{k=1}^{J} \beta_{k} \theta e_{k}$, where $\left(e_{j}\right)_{j \in\{1, \ldots, J\}}$ denote the eigenfunctions of $L_{\rho}$ such that $F_{<N}=\operatorname{span}\left(e_{j}, j \in\{1, \ldots, J\}\right)$ and $\beta=\left(\beta_{1}, \ldots, \beta_{J}\right) \in \mathbb{C}^{J}$. The condition $f_{\epsilon}+h \in \mathcal{D}_{N}$ reads $\left\langle h, e_{j}\right\rangle_{L^{2}(\rho)}=\alpha_{j}$ with $\alpha_{j}=-\left\langle f_{\epsilon}, e_{j}\right\rangle_{L^{2}(\rho)}=O(\epsilon)$. Denoting $\beta=\left(\beta_{1}, \ldots, \beta_{J}\right)$ and $\langle u, v\rangle_{Q, \rho \theta}=\int_{Q} u(x) \bar{v}(x) \rho(x) \theta(x) d x$, this is equivalent to $M \beta=\alpha$ where $M$ is the $J \times J$ matrix $M=\left(\left\langle e_{j}, e_{k}\right\rangle_{Q, \rho \theta}\right)_{j, k=1, \ldots, J}$.

We claim that $\langle,\rangle_{Q, \rho \theta}$ is definite positive on $F_{<N}$. If not, there will exist a non zero function $v \in F_{<N}$ such that $\int_{Q}|v(x)|^{2} \rho(x) \theta(x) d x=0$. This implies that $v(x)=0$ on the non void open set $\theta(x)>0$. Since $v$ satisfies $\Pi_{j<N}\left(L_{\rho}-\nu_{j}\right) v=0$, the uniqueness theorem for second order elliptic operators implies $v(x)=0$ for all $x \in \Omega$. As a consequence, the matrix $M$ is invertible, so that $\beta=M^{-1} \alpha=O(\epsilon)$. Hence $\|h\|_{H^{1}}=O(\epsilon)$. The proof of our lemma is complete.

We are now in position to achieve the proof of Theorem $\frac{\operatorname{thm} 2}{1.2}$. We first observe that if $\nu_{h m \in} \in[0, M]$ and $\psi_{h} \in L^{2}(\rho)$ satisfy $\left\|\psi_{h}\right\|_{L^{2}}=1, h^{-2} Q_{h} \psi_{h}=\nu_{h} \psi_{h}$, then thanks to Lemma 2.2 the family $\left(\psi_{h}\right)_{h \in] 0,1]}$ is relatively compact in $L^{2}(\rho)$ so that we can suppose (extracting a subsequence $h_{k}$ ) that $\nu_{h} \rightarrow \nu$ and $\psi_{h} \rightarrow \psi$ in $L^{2}(\rho),\|\psi\|_{L^{2}}=1$, and moreover by Lemma $\frac{12.2}{2.2}$, the limit $\psi$ belongs to $H^{1}(\rho)$. Given $\theta \in \mathcal{D}_{0}$, it follows from self-ajointness of $Q_{h}$ and Lemma 3 3.1 that

$$
0=\left\langle\left(h^{-2} Q_{h}-\nu_{h}\right) \psi_{h}, \theta\right\rangle_{L^{2}(\rho)}=\left\langle\psi_{h},\left(L_{\rho}-\nu_{h}\right) \theta\right\rangle_{L^{2}(\rho)}+O\left(h^{1 / 2}\right)
$$

Making $h \rightarrow 0$ we obtain $\left\langle\psi,\left(L_{\rho}-\nu\right) \theta\right\rangle_{L^{2}(\rho)}=0$ for all $\theta \in \mathcal{D}_{0}$. It follows that $\left(L_{\rho}-\nu\right) \psi=0$ in the distribution sense, and integrating by parts that $\partial_{n} \psi$ vanish on $\Gamma_{\text {reg }}$. Since $\psi \in$ $H^{1}(\rho)$, we get as above using $\frac{11 \mathrm{ug}}{1.16}$ that $\partial_{n} \psi \overline{\overline{1}} .5$, and it follows that $\psi \in D\left(L_{\rho}\right)$. This shows that $\nu$ is an eigenvalue of $L_{\rho}$, and thus (II.19) is satisfied. Moreover, by compactness in $L^{2}$ of the sequence $\psi_{h}$, one gets that for any $\epsilon>0$ small enough, there exists $h_{\epsilon}>0$ such that

$$
\sharp \operatorname{Spec}\left(h^{-2} Q_{h}\right) \cap\left[\nu_{j}-\epsilon, \nu_{j}+\epsilon\right] \leq m_{j}
$$

for $\left.h \in] 0, h_{\epsilon}\right]$ with $h_{\epsilon}>0$ small enough. It remains to show that there is equality in $\frac{p p m}{3.17}$, and we shall proceed by induction on $j$.

Let $\epsilon>0$ small be given such that for $0 \leq \nu_{j} \leq M+1$, the intervals $I_{j}^{\epsilon}=\left[\nu_{j}-\epsilon, \nu_{j}+\epsilon\right]$ are disjoint. Let $\left(\mu_{j}\right)_{j \geq 0}$ the increasing sequence of eigenvalues of $h^{-2} Q_{h}, \sigma_{N}=\sum_{j=1}^{N} m_{j}$ and $\left(e_{k}\right)_{k \geq 0}$ the eigenfunctions of $L_{\rho}$ such that for all $k \in\left\{1+\sigma_{N}, \ldots, \sigma_{N+1}\right\}$, one has $\left(L_{\rho}-\nu_{N+1}\right) e_{k}=0$. As 0 is a simple eigenvalue of both $L_{\rho}$ and $Q_{h}$, we have clearly $\nu_{0}=\mu_{0}=0$ and $m_{0}=1=\sharp \operatorname{Spec}\left(h^{-2} Q_{h}\right) \cap\left[\nu_{0}-\epsilon, \nu_{0}+\epsilon\right]$.

Suppose that for all $n \leq N, m_{n}=\sharp \operatorname{Spec}\left(h^{-2} Q_{h}\right) \cap\left[\nu_{n}-\epsilon, \nu_{n}+\epsilon\right]$. Then, one has by 1.59 , for $h \leq h_{\varepsilon}$

$$
\mu_{1+\sigma_{N}} \geq \nu_{N+1}-\epsilon
$$


By min-max principle, if $G$ is a finite dimensional subspace of $H^{1}$ with $\operatorname{dim}(G)=1+\sigma_{N+1}$, one has

$$
\mu_{\sigma_{N+1}} \leq \sup _{\psi \in G,\|\psi\|=1}\left\langle h^{-2} Q_{h} \psi, \psi\right\rangle_{L^{2}(\rho)}
$$

Thanks to Lemma $\frac{1 \mathrm{lem} 6}{3.2}$ for all $e_{k}, 0 \leq k \leq \sigma_{N+1}$ and all $\alpha>0$, there exists $e_{k, \alpha} \in \mathcal{D}_{0}$ such that $\left\|e_{k}-e_{k, \alpha}\right\|_{H_{\rho}^{1}} \leq \alpha$. Let $G_{\alpha}$ be the vector space span by the $e_{k, \alpha}, 0 \leq k \leq \sigma_{N+1}$. For $\alpha$ small enough, one has $\operatorname{dim}\left(G_{\alpha}\right)=1+\sigma_{N+1}$. From Lemma $\frac{1 \text { lem5 }}{3.1,}$ one has

$$
\left\langle h^{-2} Q_{h} e_{k, \alpha}, e_{k^{\prime}, \alpha}\right\rangle_{L^{2}(\rho)}=\left\langle L_{\rho} e_{k, \alpha}, e_{k^{\prime}, \alpha}\right\rangle_{L^{2}(\rho)}+O_{\alpha}\left(h^{1 / 2}\right)
$$

Since $e_{k, \alpha} \in \mathcal{D}_{0}$, one has $\left\langle L_{\rho} e_{k, \alpha}, e_{k^{\prime}, \alpha}\right\rangle_{L^{2}(\rho)}=\frac{\alpha_{d}}{2}\left\langle\nabla e_{k, \alpha}, \nabla e_{k^{\prime}, \alpha}\right\rangle_{L_{\rho}^{2}}$, and $\left\langle\nabla e_{k, \alpha}, \nabla e_{k^{\prime}, \alpha}\right\rangle_{L_{\rho}^{2}}=$ $\left\langle\nabla e_{k}, \nabla e_{k^{\prime}}\right\rangle_{L_{\rho}^{2}}+O(\alpha)$. Therefore, for $\psi \in G_{\alpha},\|\psi\|=1$, we get

$$
\left\langle h^{-2} Q_{h} \psi, \psi\right\rangle_{L^{2}(\rho)} \leq \nu_{N+1}+C \alpha+O_{\alpha}\left(h^{1 / 2}\right)
$$

Taking $\alpha>0$ small enough and $h<h_{\alpha}$ we obtain from $\frac{\min 2}{3.19}, \frac{\min 3}{3.21} \mu_{\sigma_{N+1}} \leq \nu_{N+1}+\epsilon$. Combining this with $\frac{\text { min1 }}{3.18}$ and $\frac{\text { ppm }}{3.17}$, we get $m_{N+1}=\sharp \operatorname{Spec}\left(h^{-2} Q_{h}\right) \cap\left[\nu_{N+1}-\epsilon, \nu_{N+1}+\epsilon\right]$.

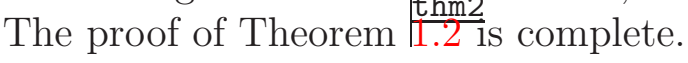

\section{Application to random placement of non-overlapping balls}

In this section, we suppose that $\Omega$ is a bounded Lipschitz stratified connected open subset of $\mathbb{R}^{d}$ with $d \geq 2$. Let $N \in \mathbb{N}, N \geq 2$ and $\epsilon>0$ be given. Let $\mathcal{O}_{N, \epsilon}$ be the open bounded subset of $\mathbb{R}^{N d}$

$$
\mathcal{O}_{N, \epsilon}=\left\{x=\left(x_{1}, \ldots, x_{N}\right) \in \Omega^{N}, \forall 1 \leq i<j \leq N,\left|x_{i}-x_{j}\right|>\epsilon\right\}
$$

We introduce the kernel

$$
K_{h}(x, d y)=\frac{1}{N} \sum_{j=1}^{N} \delta_{x_{1}} \otimes \ldots \otimes \delta_{x_{j-1}} \otimes h^{-d} \varphi\left(\frac{x_{j}-y_{j}}{h}\right) d y_{j} \otimes \delta_{x_{j+1}} \otimes \ldots \otimes \delta_{x_{N}}
$$

and the associated Metropolis operator on $L^{2}\left(\mathcal{O}_{N, \epsilon}\right)$

$$
T_{h}(u)(x)=m_{h}(x) u(x)+\int_{O_{N, \epsilon}} u(y) K_{h}(x, d y)
$$

with

$$
m_{h}(x)=1-\int_{O_{N, \epsilon}} K_{h}(x, d y) .
$$

The operator $T_{h}$ is Markov and self -adjoint on $L^{2}\left(\mathcal{O}_{N, \epsilon}\right)$. The configuration space $\mathcal{O}_{N, \epsilon}$ is the set of $N$ disjoints closed balls of radius $\epsilon / 2$ in $\mathbb{R}^{d}$, with centers at the $x_{j} \in \Omega$. The topology of this set, and the geometry of its boundary is in general hard to understand (references a trouver), but since $d \geq 2, \mathcal{O}_{N, \epsilon}$ is clearly non void and connected for a given $N$ if $\epsilon$ is small enough. The metropolis kernel $T_{h}$ is associated to the following algorithm: at each step, we choose uniformly at random a ball, and we move it center 
uniformly at random in $\mathbb{R}^{d}$ in a ball of radius $h$. If the new configuration is in $\mathcal{O}_{N, \epsilon}$, the change is made. Otherwise, the configuration is kept as it started.

In order to study the random walk associated to $T_{h}$ in in Proposition prop7 ${ }_{1} e_{\text {plemplo }}$ prove that the open set $\mathcal{O}_{N, \epsilon}$ is Lipschitz stratified for $\epsilon>0$ small enough, and in lemma $\frac{1 \text { i.m we }}{4.5}$ prove that the kernel of the iterated operator $T_{h}^{M}$ (with $M$ large, but independent of $h$ ) admits a suitable lower bound, so that we will be able to use our remark $\frac{\text { rem2 }}{2.6 .}$

We define $\Gamma_{\text {reg }}$ and $\Gamma_{\text {sing }}$ the set of regular and singular points of $\partial \mathcal{O}_{N, \epsilon}$ as follows. Let us denote $\mathbb{N}_{N}=\{1, \ldots, N\}$. For $x \in \overline{\mathcal{O}}_{N, \epsilon}$ we set

$$
\begin{aligned}
& R(x)=\left\{i \in \mathbb{N}_{N}, x_{i} \in \partial \Omega\right\} \\
& S(x)=\left\{\tau=\left(\tau_{1}, \tau_{2}\right) \in \mathbb{N}_{N}, \tau_{1}<\tau_{2} \text { and }\left|x_{\tau_{1}}-x_{\tau_{2}}\right|=\epsilon\right\} \\
& r(x)=\sharp R(x), s(x)=\sharp S(x)
\end{aligned}
$$

The functions $r$ and $s$ are lower semi-continuous and any $x \in \overline{\mathcal{O}}_{N, \epsilon}$ belongs to $\partial \mathcal{O}_{N, \epsilon}$ iff $r(x)+s(x) \geq 1$. We define

$$
\begin{aligned}
\Gamma_{r e g}=\left\{x \in \overline{\mathcal{O}}_{N, \epsilon}, s(x)=1 \text { and } r(x)\right. & =0\} \\
\cup\left\{x \in \overline{\mathcal{O}}_{N, \epsilon}, s(x)\right. & \left.=0, R(x)=\left\{j_{0}\right\} \text { and } x_{j_{0}} \in \partial \Omega_{r e g}\right\}
\end{aligned}
$$

and $\Gamma_{\text {sing }}=\partial \mathcal{O}_{N, \epsilon} \backslash \Gamma_{\text {reg }}$. Then, $\Gamma_{\text {sing }}$ is clearly close, and the $\Gamma_{\text {reg }}$ is the reunion of smooth disjoint hypersurfaces in $\mathbb{R}^{N d}$.

prop7 Proposition 4.1 For $\epsilon>0$ small enough, the set $\mathcal{O}_{N, \epsilon}$ is connected, Lipschitz and stratified.

Proof. For $\nu \in S^{p-1}, p \geq 1$ and $\left.\delta \in\right] 0,1[$ we denote

$$
\Gamma_{ \pm}(\nu, \delta)=\left\{\xi \in \mathbb{R}^{p}, \pm\langle\xi, \nu\rangle>(1-\delta)|\xi|,|\langle\xi, \nu\rangle|<\delta\right\}
$$

We remark also that an open set $\mathcal{O} \subset \mathbb{R}^{p}$ is Lipschitz if it satisfies the cone property: $\forall a \in \partial \mathcal{O}, \exists \delta>0, \exists \nu_{a} \in S^{p-1}, \forall b \in B(a, \delta) \cap \partial \mathcal{O}$ we have

$$
b+\Gamma_{+}\left(\nu_{a}, \delta\right) \subset \mathcal{O} \text { and } b+\Gamma_{-}\left(\nu_{a}, \delta\right) \subset \mathbb{R}^{p} \backslash \overline{\mathcal{O}} .
$$

Let $\bar{x} \in \partial \mathcal{O}_{N, \epsilon}$. The equivalence relation $i \simeq j$ iff $\bar{x}_{i}$ and $\bar{x}_{j}$ can be connected by a path lying in the union of the closed balls, give us a partition $\{1, \ldots, N\}=\cup_{k=1}^{r} F_{k}$ such that

$$
\begin{aligned}
& \forall k \neq l, \forall i \in F_{k}, \forall j \in F_{l},\left|\bar{x}_{i}-\bar{x}_{j}\right|>\epsilon \\
& \forall k, \forall i \neq j \in F_{k}, \exists\left(n_{l}\right) \in F_{k}, 1 \leq l \leq m, n_{1}=i, n_{m}=j,\left|\bar{x}_{n_{l}}-\bar{x}_{n_{l+1}}\right|=\epsilon .
\end{aligned}
$$

Observe that in the case where $\sharp F_{k}=1$ the second condition is empty. We look for $\nu \in S^{N d-1}$ such that the cone property at $\bar{x}$ holds with $\nu$. We construct the coordinates of $\nu$ according to the partition $\left(F_{k}\right)_{k}$. Let $k \in\{1, \ldots, r\}$.

Suppose that $F_{k}=\left\{j_{k}\right\}$ for some $j_{k} \in\{1, \ldots, N\}$. If $\bar{x}_{j_{k}} \in \Omega$, we set $\xi_{j_{k}}=0$. If $\bar{x}_{j_{k}} \in \partial \Omega$, thanks to the cone property satisfied by $\Omega$ we can find $\xi_{j_{k}} \in S^{d-1}$ and $\delta_{k}>0$ such that

$$
\bar{x}_{j_{k}}+\Gamma_{+}\left(\xi_{j_{k}}, \delta_{k}\right) \subset \Omega \text { and } \bar{x}_{j_{k}}+\Gamma_{-}\left(\xi_{j_{k}}, \delta_{k}\right) \subset \mathbb{R}^{d} \backslash \bar{\Omega}
$$


Suppose that $\sharp F_{k} \geq 2$. If $\bar{x}_{j} \in \Omega$ for all $j \in F_{k}$ we fix $y_{k} \in \mathbb{R}^{d}$ and for all $j \in F_{k}$ we set $\xi_{j}=\bar{x}_{j}-y_{k}$. Hence $x_{j}(t)=\bar{x}_{j}+t \xi_{j}$ is obtained from $\bar{x}_{j}$ by homothety centered in $y_{k}$, with ratio $1+t$. Consequently, for all $i, j \in F_{k}$ and $t$ close to 0 , we have $\left|x_{i}(t)-x_{j}(t)\right|>\epsilon$ for $t>0$ and there exists at least two distinct indices $i, j \in F_{k}$ such that $\left|x_{i}(t)-x_{j}(t)\right|<\epsilon$ for $t<0$.

If there exists $i_{0} \in F_{k}$ such that $\bar{x}_{i_{0}} \in \partial \Omega$, the cone property provides us $\eta_{i_{0}} \in S^{d-1}$ and $\delta_{i_{0}}>0$. Moreover, taking $\epsilon>0$ sufficiently small, we can suppose that all the $\bar{x}_{i}, i \in F_{k}$ are close to $\bar{x}_{i_{0}}$, since $\left|\bar{x}_{i}-\bar{x}_{i_{0}}\right| \leq \epsilon(N-1)$. Thus, for all $i \in F_{k}$, we have $b+\Gamma_{+}\left(\eta_{i_{0}}, \delta_{i_{0}}\right) \subset \Omega$ for all $b \in \bar{\Omega},\left|b-\bar{x}_{i}\right| \leq \delta_{i_{0}}$. Let $y_{i_{0}}(z)=\bar{x}_{i_{0}}-z$

$e t a_{i_{0}}$ and for $i \in F_{k}, \xi_{i}^{\prime}(z)=\bar{x}_{i}-y_{i_{0}}(z)$. Then, for $z>0$ large enough and $\alpha>0$ sufficiently small, we have $\xi_{i}=\alpha \xi_{i}^{\prime}(z) \in \Gamma\left(\eta_{i_{0}}, \delta_{i_{0}}\right)$. In particular, for $i \in F_{k}$ and $t>0$ close to $0, \bar{x}_{i}+t \xi_{i}$ belongs to $\Omega$, while for $t<0$, one has $\bar{x}_{i_{0}}+t \xi_{i_{0}} \notin \bar{\Omega}$. Moreover, the same argument as above shows that for $i \neq j$, the functions $t \mapsto\left|\bar{x}_{i}-\bar{x}_{j}+t\left(\xi_{i}-\xi_{j}\right)\right|$ are strictly increasing near $t=0$.

Observe that $\xi=\left(\xi_{1}, \ldots, \xi_{N}\right) \neq 0$, since if $\xi_{i}=0$ for all $i$, then $\sharp F_{k}=1$ and $\bar{x}_{j_{k}} \in \Omega$ for all $k$ which contradicts $\bar{x} \in \partial \mathcal{O}_{N, \epsilon}$. Finally, we take $\nu=\frac{\xi}{|\xi|}$. Then for $\delta>0$ small enough, the cone property is satisfied at $\bar{x}$ with $(\nu, \delta)$. Thus $\mathcal{O}_{N, \epsilon}$ is Lipschitz.

Let us show that $\mathcal{O}_{N, \epsilon}$ is connected for $\epsilon$ small enough. We define for $j \in \mathbb{N}_{N}$ the two applications $\pi_{j}$ from $\mathbb{R}^{N d}$ to $\mathbb{R}^{N d}$ and $\sigma_{j}$ from $\mathbb{R}^{d}$ to $\mathbb{R}^{N d}$ by

$$
\begin{array}{r}
\pi_{j}\left(x_{1}, \ldots, x_{j}, \ldots, x_{N}\right)=\left(x_{1}, \ldots, 0, \ldots, x_{N}\right) \\
\sigma_{j}(y)=(0, \ldots, y, \ldots, 0)
\end{array}
$$

so that $x=\pi_{j}(x)+\sigma_{j}\left(x_{j}\right)$. For $F \subset \mathbb{N}_{N}$ we define $\sigma_{F}: \Pi_{j \in F} \mathbb{R}^{d} \rightarrow \mathbb{R}^{N d}$ by $\sigma_{F}(x)=$ $\sum_{j \in F} \sigma_{j}\left(x_{j}\right)$. We have the following geometric lemma.

lem7ter Lemma 4.2 There exists $\epsilon_{0}>0$ and $\delta_{0}>0$ such that for all $\left.\left.\epsilon \in\right] 0, \epsilon_{0}\right]$ and all $n \in \mathbb{N}_{N}$, there exists a finite covering $\left(U_{l}\right)_{l}$ of $\mathcal{O}_{N, \epsilon}$ such that for all l there exists a subset $F_{n} \subset \mathbb{N}_{N}$ with $\sharp F_{n}=n$, there exists $\nu \in S^{n d-1}$ such that

$$
\forall x \in U_{l} \cap \mathcal{O}_{N, \epsilon}, x+\sigma_{F_{n}}\left(\Gamma_{+}\left(\nu, \delta_{0}\right)\right) \subset \mathcal{O}_{N, \epsilon}
$$

Moreover, there exists $c_{0}>0$ such that for all $k, l \in F_{n}$ with $k \neq l$ and for $t \in\left[0, \delta_{0}\right]$, we have

$$
\forall x \in U_{l} \cap \mathcal{O}_{N, \epsilon},\left|x_{k}+t \nu_{k}-x_{l}-t \nu_{l}\right|^{2} \geq \epsilon^{2}+c_{0} \epsilon t
$$

Proof. This lemma means that we can select an arbitrary number of balls $n$, and that moving only these balls by a vector in $\Gamma_{+}\left(\nu, \delta_{0}\right)$ while keeping the other balls fixed, results in an admissible configuration. We shall proceed by induction on $N \geq 1$. For $N=1$, this is true since $\Omega$ is Lipschitz. Let $N \geq 2$ and suppose that the property is true until rank $N-1$ and let $\tilde{\epsilon}_{0}$ be the corresponding parameter. For $\left.\epsilon \in\right] 0, \tilde{\epsilon}_{0}[$ and $\beta>0$, we have the partition

$$
\mathcal{O}_{N, \epsilon}=\mathcal{U}_{N, \epsilon, \beta} \cup \mathcal{V}_{N, \epsilon, \beta}
$$

with $\mathcal{U}_{N, \epsilon, \beta}=\left\{x \in \mathcal{O}_{N, \epsilon}, \sup _{i \neq j}\left|x_{i}-x_{j}\right|<\epsilon+\beta\right\}$. Using the induction hypothesis, it is easy to see that for any $\beta>0$, there exists $\tilde{\epsilon}_{1}(\beta)<\tilde{\epsilon}_{0}$ and $\tilde{\delta}_{0}(\beta)>0$ such that the conclusion of the Lemma holds true on $\left.\left.\mathcal{V}_{N, \epsilon, \beta}, \forall \epsilon \in\right] 0, \tilde{\epsilon}_{1}(\beta)\right]$. Hence it remains to find a 
suitable covering of $\mathcal{U}_{N, \epsilon, \beta}$. For any $\alpha>0$, choosing $\beta$ and $\tilde{\epsilon}_{1}$ small enough we can suppose that

$$
\mathcal{U}_{N, \epsilon, \beta} \subset \cup_{y^{0} \in F_{\alpha}} B\left(y^{0}, \alpha\right)^{N}
$$

for some finite set $F_{\alpha} \subset \Omega$. Moreover, since $\Omega$ is Lipschitz, we can suppose that $\alpha$ is sufficiently small so that

$$
\exists \delta_{0}^{\prime}>0, \forall y^{0} \in F_{\alpha}, \exists v\left(y^{0}\right) \in S^{d-1}, \forall y \in B\left(y^{0}, \alpha\right), y+\Gamma_{+}\left(v\left(y^{0}\right), 2 \delta_{0}^{\prime}\right) \subset \Omega .
$$

Moreover, we can suppose that $\delta_{0}^{\prime}$ is sufficiently small so that the following holds true:

$$
\begin{aligned}
& \forall v, u_{1}, \ldots, u_{N} \in S^{d} \text {, s.t. } \forall j,\left\langle v, u_{j}\right\rangle=0, \exists v^{\prime} \in \Gamma_{+}\left(v, 2 \delta_{0}^{\prime}\right), \\
& \forall \xi \in \Gamma_{+}\left(v^{\prime}, \delta_{0}^{\prime}\right), \forall j,\left\langle\xi, u_{j}\right\rangle \neq 0 .
\end{aligned}
$$

Indeed, the set $A=\left\{v \in S^{d},\left\langle v, u_{j}\right\rangle=0, \forall j\right\}$ is contained in a finite union of equators and thus ( $(4.16)$ is obvious by taking $v^{\prime}$ close to $v$ in the complementary.

Condition ( $\left(\begin{array}{l}1.15 \\ 1.15\end{array}\right)$ gives us a critical value for $\tilde{\epsilon}_{1}$ and we now suppose that $\left.\left.\epsilon \in\right] 0, \tilde{\epsilon}_{1}\right]$. By compactness, it remains to show that $y_{0} \in F_{\alpha}$ being fixed the following property holds true:

$$
\begin{aligned}
& \forall x^{0} \in B\left(y^{0}, \alpha\right)^{N} \cap \mathcal{O}_{N, \epsilon}, \forall n \in \mathbb{N}_{N}, \exists F_{n} \subset \mathbb{N}_{N} \text { s.t. } \sharp F_{n}=n, \exists \nu \in S^{n d-1}, \exists r>0 \text {, s.t. } \\
& \forall x \in B\left(x^{0}, r\right) \cap \mathcal{O}_{N, \epsilon}, \forall \xi \in \Gamma_{+}\left(\nu, \delta_{0}^{\prime}\right), \forall k \neq j,\left|\left(x+\sigma_{F}(\xi)\right)_{j}-\left(x+\sigma_{F}(\xi)\right)_{k}\right|>\epsilon
\end{aligned}
$$

Let $x^{0} \in B\left(y^{0}, \alpha\right)^{N}$ and $n \in \mathbb{N}_{N}$ being fixed. We construct $F_{n}$ and $\nu \in S^{n d-1}$ by induction on $n$. We look for $\nu$ under the form $\nu=\lambda(v,(1-1 / N) v, \ldots,(1-(n-1) / N) v)$, where $v \in S^{d-1}$ and $\lambda$ is a normalizing constant. We claim that we can find $F_{n}=\left\{j_{1}, \ldots j_{n}\right\}$ and $v \in S^{d-1}$ such that

$$
\left\langle v, x_{j_{1}}^{0}\right\rangle>\ldots>\left\langle v, x_{j_{n}}^{0}\right\rangle>\left\langle v, x_{s}^{0}\right\rangle, \forall s \notin F_{n}
$$

If $n=1$ we denote $F^{\prime}$ the set of index $i$ such that the map $s \in \mathbb{N}_{N} \mapsto\left\langle v\left(y^{0}\right), x_{s}^{0}\right\rangle$ has a maximum in $s=i$. If $\sharp F^{\prime}=1$ then we can take $\left(F_{1}, v\right)=\left(F^{\prime}, v\left(y^{0}\right)\right)$. If $\sharp F^{\prime} \geq 2$, thanks to $\left(\frac{s_{1}^{13}}{4.17}\right)$ we can find $v$ close to $v\left(y^{0}\right)$ such that $s \mapsto\left\langle v, x_{s}^{0}\right\rangle$ has a unique maximum for some $s=j_{1}$ and we set $F_{1}=\left\{j_{1}\right\}$.

Suppose now that $n \geq 1$ and that $\left(F_{n}, v\right)$ satisfies ( $\left(\frac{s 14}{4.18}\right)$. Let $F^{\prime \prime}$ be the set of index $i \notin F_{n}$ such that the map $s \notin F_{n} \mapsto\left\langle v, x_{s}^{0}\right\rangle$ has a maximum in $s=i$. If $\sharp F^{\prime \prime}=1$ then $\left(F_{n+1}, v\right)=\left(F_{n} \cup F^{\prime \prime}, v\right)$ satisfies the expected property. If $\sharp F^{\prime \prime} \geq 2$ we can find $v^{\prime \prime}$ close to $v$ so that we still have

$$
\left\langle v^{\prime \prime}, x_{j_{1}}^{0}\right\rangle>\ldots>\left\langle v^{\prime \prime}, x_{j_{n}}^{0}\right\rangle>\left\langle v^{\prime \prime}, x_{s}^{0}\right\rangle, \forall s \notin F_{n}
$$

and additionally $\left\langle x_{k}, v^{\prime \prime}\right\rangle \neq\left\langle x_{l}, v^{\prime \prime}\right\rangle$ for all $k, l \in F^{\prime \prime}$ with $k \neq l$, and $\left\langle x_{k}, v^{\prime \prime}\right\rangle>\left\langle x_{s}, v^{\prime \prime}\right\rangle$ for all $k \in F^{\prime \prime}, s \notin F_{n_{1}} \cup F^{\prime \prime}$. This permits to find easily $j_{n+1} \in F^{\prime \prime}$ such that setting $F_{n+1}=F_{n} \cup\left\{j_{n+1}\right\},($ (S.18) holds true at rank $n+1$.

We turn back to the proof of $\left(\frac{1}{1.17}\right)$. Let $x \in B\left(x^{0}, r\right)$ and $\xi=\left(\xi_{j_{1}}, \ldots, \xi_{j_{n}}\right) \in \Gamma_{+}\left(\nu, \delta_{0}^{\prime}\right)$ with $\delta_{0}^{\prime}$ given by the above construction and $r>0$ to be chosen small enough. We denote $\nu=\left(\nu_{j_{1}}, \ldots, \nu_{j_{n}}\right)$ so that $\nu_{j_{k}}=\lambda\left(1_{1}-(k-1) / N\right) v$. The fact that $x+\sigma_{F_{n}}(\xi) \in \Omega^{N}$ is a straightforward consequence of $\left(\frac{14.15}{4.15}\right)$ and it remains to show that the distance between two different balls remains bounded from below by $\epsilon$. Let $j, k \in \mathbb{N}_{N}$ with $j \neq k$. If $j, k \notin F_{n}$, there is nothing to prove. 
In the case where $j, k \in F_{n}$, we can write $j=j_{p}$ and $k=j_{q}$ with $p<q$ and we have

$$
\begin{aligned}
\mid\left(x+\sigma_{F}(\xi)\right)_{j}- & \left.\left(x+\sigma_{F}(\xi)\right)_{k}\right|^{2} \geq \epsilon^{2}+2\left\langle x_{j_{p}}-x_{j_{q}}, \xi_{j_{p}}-\xi_{j_{q}}\right\rangle \\
\geq \epsilon^{2}+2|\xi|\left\langle x_{j_{p}}^{0}-x_{j_{q}}^{0}, \nu_{j_{p}}-\nu_{j_{q}}\right\rangle-4 r\left(\left|\xi_{j_{p}}\right|+\left|\xi_{j_{q}}\right|\right) & \\
- & 2|\xi|\left|x_{j_{p}}^{0}-x_{j_{q}}^{0}\right|\left(\left|\frac{\xi_{j_{p}}}{|\xi|}-\nu_{j_{p}}\right|+\left|\frac{\xi_{j_{q}}}{|\xi|}-\nu_{j_{q}}\right|\right) \\
\geq \epsilon^{2}+ & 2 \frac{\lambda}{N}(q-p)|\xi|\left\langle v, x_{j_{p}}^{0}-x_{j_{q}}^{0}\right\rangle-8 r|\xi|-4|\xi| \delta_{0}^{\prime}\left|x_{j_{p}}^{0}-x_{j_{q}}^{0}\right|
\end{aligned}
$$

Thanks to $\left(\frac{\mid \mathbf{s} 14}{4.18}\right),\left\langle v, x_{j_{p}}^{0}-x_{j_{q}}^{0}\right\rangle>c_{1}\left|x_{j_{p}}^{0}-x_{j_{q}}^{0}\right|$, for some $c_{1}>0$ independant on $\epsilon$. Hence, taking $\left.\left.\delta_{0}^{\prime} \in\right] 0, \lambda c_{1} /(8 N)\right]$ and $\left.\left.r \in\right] 0, \lambda \epsilon /(16 N)\right]$, we get

$$
\left|\left(x+\sigma_{F}(\xi)\right)_{j}-\left(x+\sigma_{F}(\xi)\right)_{k}\right|^{2}>\epsilon^{2}+c_{1} \frac{\lambda \epsilon}{N}|\xi|
$$

so that $\left(\frac{\$ 100}{4.11}\right)$ is satisfied. Moreover, as $\lambda$ is of order $n^{-1 / 2}$, $\left(\frac{\$ 10}{4.12}\right)$ holds true with $c_{0}=\frac{c_{1}}{N \sqrt{n}}$.

In the case $j \in F_{n}$ and $k \notin F_{n}$, similar computation leads to the same result. Taking $\delta_{0}=\min \left(\tilde{(} \delta_{0}(\beta), \delta_{0}^{\prime}\right)$, the proof of the lemma is complete.

sublemma1 Lemma 4.3 There exists $\epsilon_{1}>0$ and $m \geq 1$ such that $\left.\left.\forall \epsilon \in\right] 0, \epsilon_{1}\right], \forall x, y \in \mathcal{O}_{N, m \epsilon}$ satisfiying $\inf _{j, k}\left|x_{j}-y_{k}\right|>m \epsilon$, there exists a continuous path $\gamma:[0,1] \rightarrow \mathcal{O}_{N, \epsilon}$ such that $\gamma(0)=x$ and $\gamma(1)=y$.

Proof. For $z^{\prime}=\left(z_{2}, \ldots, z_{N}\right) \in \Omega^{N-1}$ and $k \geq 1$ we denote $\tilde{\Omega}_{k}\left(z^{\prime}\right)=\Omega \backslash \cup_{j=2}^{N} \bar{B}\left(z_{j}, k \epsilon\right)$. We claim that there exists $\epsilon_{1}>0$ such that for $\left.\left.\epsilon \in\right] 0, \epsilon_{1}\right]$ and $m \geq 1$ large enough, the following property holds true:

$$
\forall x^{\prime} \in \mathcal{O}_{N-1, m \epsilon}, \forall u, v \in \tilde{\Omega}_{m}\left(x^{\prime}\right), \exists \gamma \in C\left([0,1], \tilde{\Omega}_{1}\left(x^{\prime}\right)\right), \text { s.t. } \gamma(0)=u, \gamma(1)=v
$$

Indeed, $\Omega$ being connected, for any $u, v \in \tilde{\Omega}_{m}\left(x^{\prime}\right)$ there exists a continous path $\gamma:[0,1] \rightarrow$ $\Omega$ from $u$ to $v$. Moreover, using the fact that $\partial \Omega$ is Lipschitz and taking $m$ sufficiently large, we can modify the path $\gamma$ in a path $\tilde{\gamma}$ that avoids the balls $\bar{B}\left(x_{j}, \epsilon\right)$ and remains in $\Omega$.

Now, let $x, y \in \mathcal{O}_{N, m \epsilon}$ with $\inf _{j, k}\left|x_{j}-y_{k}\right|>m \epsilon$. Thanks to $\left(\frac{\$ 15}{1.22}\right)$, we can find a continous path from $x$ to $\left(y_{1}, x_{2}, \ldots, x_{N}\right)$ with values in $\mathcal{O}_{N, \epsilon}$. As $\left|y_{1}-x_{j}\right|>\lambda$ for all $j$, we can apply $\left(\frac{515}{4.22}\right)$ with $z^{\prime}=\left(y_{1}, x_{3}, \ldots, x_{N}\right)$ so that we can find a continuous path in $\mathcal{O}_{N, \epsilon}$ joining $\left(y_{1}, x_{2}, \ldots, x_{N}\right)$ and $\left(y_{1}, y_{2}, x_{3}, \ldots, x_{N}\right)$. Iterating this process we obtain a continous path from $x$ to $y$, with values in $\mathcal{O}_{N, \epsilon}$.

We are now in position to prove that $\mathcal{O}_{N, \epsilon}$ is connected for $\epsilon$ small enough. Let $\epsilon_{0}, \delta_{0}>0$ be given by Lemma 7.2 and $m, \epsilon_{1}$ be given by Lemma 4.3 . We can also decrease $\epsilon_{1}$ so that $\left.\forall \epsilon \in\right] 0, \epsilon_{1}\left[, \forall x \in \mathcal{O}_{N, \epsilon}, \exists y \in \mathcal{O}_{N, m_{\text {l }} \text { em.t. }}^{\text {Stter }} \inf _{i, j}\left|x_{i}-y_{j}\right|>2 m \epsilon\right.$. Let $\epsilon \leq \min \left(\epsilon_{1}, \epsilon_{0}, c_{0} \delta_{0} /\left(m_{\text {sublemma1 }}^{2} 1\right)\right)$ with $c_{0}$ given by Lemma $\overline{4}$. 2 .

Thanks to Lemma 4.3 , it suffices to show that for any $x \in \mathcal{O}_{N, \epsilon}$ there exists a continuous path $\gamma:[0,1] \rightarrow \mathcal{O}_{N, \epsilon}$ such that $\gamma(0)=x$ and $\gamma(1) \in \in_{\mathcal{O}, m \epsilon}$.

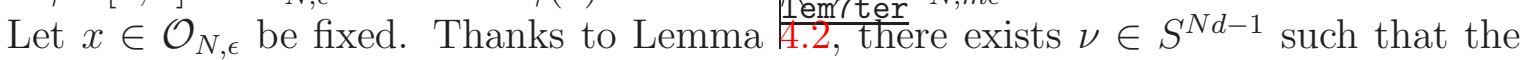
segment $x+\left[0, \delta_{0}\right] \nu$ is contained in $\Omega^{N}$ and moreover for any $k \neq l$ and any $t \in\left[0, \delta_{0}\right]$,

$$
\left|x_{k}+t \nu_{k}-x_{l}-t \nu_{l}\right|^{2} \geq \epsilon^{2}+c_{0} \epsilon t .
$$


Hence, the path $t \in[0,1] \mapsto \gamma(t)=x+t \delta_{0} \nu$ has the required properties. This achieves to prove that $\mathcal{O}_{N, \epsilon}$ is connected.

Let us now prove that $\mathcal{O}_{N, \epsilon}$ is stratified. Let $u \in H^{-1 / 2}\left(\mathcal{O}_{N, \epsilon}\right)$ be supported in $\Gamma_{\text {reg. }}$. We have to show that $u$ is identically zero. This is a local problem and we can suppose that $u$ is supported in a small open set $U \subset \mathbb{R}^{N d}$ such that $\tilde{U}:=U \cap \mathcal{O}_{N, \epsilon}=\left\{\left(x_{1}+\varphi\left(x^{\prime}\right), x^{\prime}\right), x_{1} \in\right.$ ] $0, \alpha\left[, x^{\prime} \in V\right\}, \delta U:=U \cap \partial \mathcal{O}_{N, \epsilon}=\left\{\left(\varphi\left(x^{\prime}\right), x^{\prime}\right), x^{\prime} \in V\right\}$, where $\varphi: V \subset \mathbb{R}^{N d-1} \rightarrow \mathbb{R}$ is a Lipschitz function. Denote

$$
\begin{aligned}
\kappa:] 0, \alpha[\times V & \rightarrow \tilde{U}, & k: V & \rightarrow \delta U \\
\left(x_{1}, x^{\prime}\right) & \mapsto\left(x_{1}+\varphi\left(x^{\prime}\right), x^{\prime}\right) & x^{\prime} & \mapsto\left(\varphi\left(x^{\prime}\right), x^{\prime}\right)
\end{aligned}
$$

and for $\chi \in C_{0}^{\infty}(V)$, let $\phi_{\chi}(u)$ be defined by

$$
\left\langle\phi_{\chi}(u), f\right\rangle=\left\langle u,(\chi f) \circ k^{-1}\right\rangle_{H^{-1 / 2}\left(\partial O_{N, \epsilon}\right), H^{1 / 2}\left(\partial O_{N, \epsilon}\right)}
$$

for any $f \in H^{1 / 2}\left(\mathbb{R}^{N d-1}\right)$. Then $\phi_{\chi}(u) \in H^{-1 / 2}\left(\mathbb{R}^{N d-1}\right)$ and $\operatorname{supp}\left(\phi_{\chi}(u)\right) \subset k^{-1}(\operatorname{supp}(u))$. Moreover, the distribution $\tilde{v}=\delta_{x_{1}=0} \otimes \phi_{\chi}(u)$ belongs to $H^{-1}\left(\mathbb{R}^{N d}\right)$ and $\operatorname{supp}(\tilde{v}) \subset$ $\kappa^{-1}(\operatorname{supp}(u))$.

Let $\bar{x} \in \delta U \cap \operatorname{supp}(u)$ and denote $\mathcal{D}_{N, \epsilon}=\left\{x \in\left(\mathbb{R}^{d}\right)^{N},\left|x_{i}-x_{j}\right|>\epsilon, \forall 1 \leq i<j \leq N\right\}$. Then, either $r(\bar{x})+s(\bar{x}) \geq 2$, either $\bar{x} \in \mathcal{D}_{N, \epsilon}, R(\bar{x})=\left\{j_{0}\right\}$ (say $j_{0}=1$ ) and $\bar{x}_{j_{0}} \in \partial \Omega_{\text {sing }}$. Suppose that we are in the second case and let $\chi$ be a cut-off function supported near $\bar{x}$ such that $\operatorname{supp}(\chi) \subset \mathbb{R}^{d} \times \Omega^{N-1} \cap \mathcal{D}_{N, \epsilon}$. Then, for any $\psi \in C_{0}^{\infty}\left(\bar{\Omega}^{N-1}\right)$ the linear form $u_{\psi}$ defined on $H^{1 / 2}(\partial \Omega)$ by

$$
\left\langle u_{\psi}, f\right\rangle=\left\langle\chi u, f\left(x_{1}\right) \psi\left(x_{2}, \ldots, x_{N}\right)\right\rangle_{H^{-1 / 2}(\delta U), H^{1 / 2}(\delta U)}
$$

is continuous and supported in $\partial \Omega_{\text {sing }}$. As $\partial \Omega$ is stratified, it follows that $u_{\psi}$ is equal to zero for all $\psi$ and hence, $\chi u=0$. Therefore, we can suppose that $u$ is supported in the set $\{r(x)+s(x) \geq 2\}$. For $n \in \mathbb{N}, n \geq 2$, let us introduce the following property

$$
\left(\mathcal{P}_{n}\right) \text { : for any } \bar{x} \in \delta U \text { s.t. } r(\bar{x})+s(\bar{x})=n \text {, we have } u=0 \text { near } \bar{x} \text {. }
$$

We prove this property by induction on $n$. We first assume $n=2$ and suppose that $r(\bar{x})=s(\bar{x})=1$ (the cases $r=2, s=0$ and $r=0, s=2$ are similar and left to the reader). By lower semicontinuity of the functions $r$ and $s$, for any $x \in \operatorname{supp}(u)$ close to $\bar{x}$ we have also $r(x)=s(x)=1$ and hence $R(x)=R(\bar{x})$ and $S(x)=S(\bar{x})$. Hence, we can suppose without loosing generality, that $u$ is supported in $G=\partial \Omega \times \Omega^{N-1} \cap\left\{\left|x_{i}-x_{2}\right|=\epsilon\right\}$ for some $i \in\{1,3, \ldots, N\}$. Denoting $x_{i}=\left(x_{i, 1}, \ldots, x_{i, d}\right)$ and using the fact that $\partial \mathcal{D}_{N, \epsilon}$ is invariant under any transformation of the form $x \mapsto\left(\rho\left(x_{1}\right), \ldots, \rho\left(x_{N}\right)\right)$ where $\rho$ is an affine isometry of $\mathbb{R}^{d}$, there exists a linear map $L$ on $\mathbb{R}^{N d}$ such that $L(G)$ is given by two equations

$$
\begin{aligned}
& x_{1,1}=\alpha\left(x_{1}^{\prime}\right) \\
& x_{2,1}=\beta\left(x_{2}^{\prime}, x_{i}\right) .
\end{aligned}
$$

with $\alpha$ Lipschitz and $\beta$ smooth and where $x_{j}^{\prime}=\left(x_{j, 2}, \ldots, x_{j, d}\right)$. Hence, $\nu(x)=\left(x_{1,1}-\right.$ $\left.\alpha\left(x_{1}^{\prime}\right), x_{2,1}-\beta\left(x_{2}^{\prime}, x_{i}\right), x_{1}^{\prime}, x_{2}^{\prime}, x_{3}, \ldots, x_{N}\right)$ defines a local homeomorphism of $\mathbb{R}^{N d}$ such that $\nu \circ L(G) \subset\{0\}^{2} \times \mathbb{R}^{N d-2}$. Consequently, $\tilde{w} \in H^{-1}\left(\mathbb{R}^{N d}\right)$ defined by

$$
\langle\tilde{w}, f\rangle=\langle\tilde{v}, f \circ \nu \circ L \circ \kappa\rangle
$$


satisfies $\operatorname{supp}(\tilde{w}) \subset\{0\}^{2} \times \mathbb{R}^{N d-2}$. Therefore, $\tilde{w}$ vanishes identically and hence $u$ is null near $\bar{x}$.

Suppose now that $\left(\mathcal{P}_{k}\right)$ holds for $k \leq n$ and let $\bar{x}$ be such that $r(\bar{x})+s(\bar{x})=n+1$. The lower semicontinuity of $r, s$ and the induction hypothesis show that for any $x \in \operatorname{supp}(u)$ close enough to $\bar{x}$, we have $r(x)+s(x)=n+1$ and hence $R(x)=R(\bar{x}), S(x)=S(\bar{x})$. Suppose that $r(\bar{x})=0$, then $s(\bar{x})=n+1$ and near $\bar{x}, \operatorname{supp}(u)$ is contained in $G=$ $\Omega^{N} \cap\left(\cap_{\tau \in R(\bar{x})}\left\{\left|x_{\tau_{1}}-x_{\tau_{2}}\right|=\epsilon\right\}\right.$. In particular, there exists $\sigma, \tau \in R(\bar{x})$ such that $\sigma \neq \tau$. As in the case $n=2$, we can suppose that near $\bar{x}$, the set $\{r(x)+s(x)=n+1\}$ is contained in $\left\{x_{\tau_{1}, 1}=\alpha\left(x_{\tau_{1}}^{\prime}, x_{\tau_{2}}\right), x_{\sigma_{1}, 1}=\alpha\left(x_{\sigma_{1}}^{\prime}, x_{\sigma_{2}}\right)\right\}$ for some Lipschitz functions $\alpha, \beta$ (here we forget some information). Hence, we can construct as precedently an homeomorphism $\nu$ on $\mathbb{R}^{N d}$ such that $\nu(G) \subset\{0\}^{2} \times \mathbb{R}^{N d-2}$ and the same proof as for $n=2$ still works. The cases $s=0, r=n+1$ and $s \geq 1, r_{\text {prop } f}^{>1}$ are similar and left to the reader.

The proof of proposition $\frac{\text { prop } 1 \mathrm{~S}}{1.1 \mathrm{~S}}$ complete.

remk5 Remark 4.4 Observe that in the above lemma, the smallness condition on $\epsilon$ is $N \epsilon<c$ where $c>0$ depends only on $\Omega$. The condition $N \epsilon^{d}<c$, which say that the density of the balls is small enough, does not implies that the set $\mathcal{O}_{N, \epsilon}$ has Lipschitz regularity. As an example, if $\Omega=] 0,1\left[^{2}\right.$ is the unit square in the plane, then $x=\left(x_{1}, \ldots, x_{N}\right), x_{j}=$ $((j-1) \epsilon, 0), j=1, \ldots, N$, with $\epsilon=\frac{1}{N-1}$ is a configuration point in the boundary $\partial \mathcal{O}_{N, \epsilon}$. However, $\partial \mathcal{O}_{N, \epsilon}$ is not Lipschitz at $x$ : otherwise, there will exist $\nu_{j}=\left(a_{j}, b_{j}\right)$ such that $\left(x_{1}+t \nu_{1}, \ldots, x_{N}+t \nu_{N}\right) \in \mathcal{O}_{N, \epsilon}$ for $t>0$ small enough, and this implies $a_{1}>0, a_{j+1}>a_{j}$ and $a_{N}<0$ which is impossible.

For $k \in \mathbb{N}^{*}$ we denote $B^{k}=B_{\mathbb{R}^{k}}(0,1)$ the unit euclidian ball and $\varphi_{k}(z)=\frac{1}{V o l\left(B^{k}\right)} 1_{B_{k}}(z)$.

lem7bis Lemma 4.5 Let $\epsilon$ be small. There exists $h_{0}>0, c_{0}, c_{1}>0$ and $M \in \mathbb{N}^{*}$ such that for all $\left.h \in] 0, h_{0}\right]$, one has

$$
T_{h}^{M}(x, d y)=\mu_{h}(x, d y)+c_{0} h^{-N d} \varphi_{N d}\left(\frac{x-y}{c_{1} h}\right) d y
$$

where for all $x \in \mathcal{O}_{N, \epsilon}, \mu_{h}(x, d y)$ is a positive Borel measure.

Proof. For $x, y \in \mathcal{O}_{N, \epsilon}$, we set $\operatorname{dist}(x, y)=\sup _{1 \leq i \leq N}\left|x_{i}-y_{i}\right|$. For $N \geq 1$, let us denote by $K_{h, N}$ the kernel given in 4.1 . It is sufficient to prove the following: for $\epsilon$ small, there exists $h_{0}>0, c_{0}, c_{1}>0$ and $M(N) \in \mathbb{N}^{*}$ such that for all $\left.\left.h \in\right] 0, h_{0}\right]$, one has for all non negative function $f$

$$
K_{h, N}^{M(N)}(f)(x) \geq c_{0} h^{-N d} \int_{y \in \mathcal{O}_{N, \epsilon}, \operatorname{dist}(y, x) \leq c_{1} h} f(y) d y
$$

We first remark that it is sufficient to prove the weaker version: for all $x^{0} \in \overline{\mathcal{O}}_{N, \epsilon}$, there exist $M\left(N, x^{0}\right), \alpha=\alpha\left(x^{0}\right)>0, c_{0}=c_{0}\left(x_{0}\right)>0, c_{1}=c_{1}\left(x_{0}\right)>0, h_{0}=h_{0}\left(x_{0}\right)>0$ such that for all $\left.h \in] 0, h_{0}\right]$, all $x \in \mathcal{O}_{N, \epsilon}$ and all non negative function $f$

$$
\operatorname{dist}\left(x, x^{0}\right) \leq 2 \alpha \Longrightarrow K_{h, N}^{M\left(N, x^{0}\right)}(f)(x) \geq c_{0} h^{-N d} \int_{y \in \mathcal{O}_{N, \epsilon}, \operatorname{dist}(y, x) \leq c_{1} h} f(y) d y
$$


Let us verify that $\frac{65}{4.32}$ implies $\frac{64}{4.3} 3$. Decreasing $\alpha\left(x_{0}\right)$ if necessary, we may assume that any set $\left\{\operatorname{dist}\left(x, x^{0}\right) \leq 2 \alpha\left(x_{0}\right)\right\}$ is contained in one of the open set $U_{l}$ of lemma $\frac{1 \text { em }}{4.2}$. There exists a finite set $F$ such that $\overline{\mathcal{O}}_{N, \epsilon} \subset \cup_{x^{0} \in F}\left\{\operatorname{dist}\left(x, x^{0}\right) \leq \alpha\left(x_{0}\right)\right\}$. Let $M(N)=$ $\sup _{x^{0} \in F} M\left(N, x_{0}\right), c_{i}^{\prime}=\min _{x_{0} \in F} c_{i}\left(x_{0}\right)$ and $h_{0}^{\prime}=\min _{x_{0} \in F} h_{0}\left(x_{0}\right)$. One has to check that for any $x^{0} \in F$ and any $x$ with $\operatorname{dist}\left(x, x^{0}\right) \leq \alpha\left(x^{0}\right)$, the right inequality in $\frac{g^{5}}{4.32}$ holds true with $M(N)=M\left(N, x^{0}\right)+n$ in place of $M\left(N, x^{0}\right)$, and for some constants $c_{0}, c_{1}, h_{0} \cdot$ Het $U_{l}$ be such that $\operatorname{dist}\left(x, x^{0}\right) \leq \alpha$ implies $x \in U_{l}$. Let $j$ and $\Gamma_{+}(\nu, \delta)$ be given by lemma $\frac{1}{4.2}$. Clearly, if $f$ is non negative, one has

$$
K_{h, N}^{M\left(N, x^{0}\right)+1}(f)(x) \geq \frac{1}{N} h^{-d} \int_{x+\sigma_{j}(z) \in \mathcal{O}_{N, \epsilon}} \varphi(z / h) K_{h, N}^{M\left(N, x^{0}\right)}(f)\left(x+\sigma_{j}(z)\right) d z
$$

For $\operatorname{dist}\left(x, x^{0}\right) \leq 2 \alpha\left(x^{0}\right)-c_{1}^{\prime} h / 2$, and $|z| \leq c_{1}^{\prime} h / 2, z \in \Gamma_{+}(\nu, \delta)$, one has $\operatorname{dist}(x+$ $\left.\sigma_{j}(z), x^{0}\right) \leq 2 \alpha\left(x^{0}\right)$ and by $\left.\right|_{?} ^{g} ?_{0} x+\sigma_{j}(z) \in \mathcal{O}_{N, \epsilon}$. Moreover, $\operatorname{dist}(y, x) \leq c_{1}^{\prime} h / 2 \Longrightarrow$

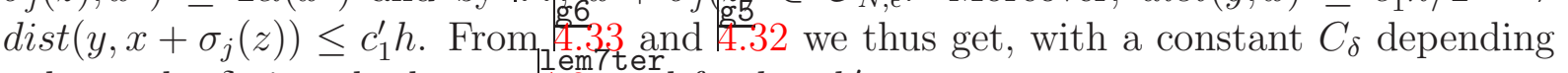

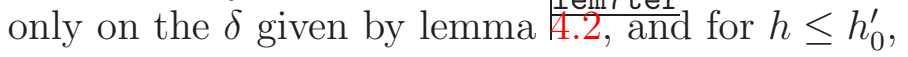

$$
\begin{aligned}
& \operatorname{dist}\left(x, x^{0}\right) \leq 2 \alpha\left(x^{0}\right)-c_{1}^{\prime} h / 2 \Longrightarrow \\
& K_{h, N}^{M\left(N, x^{0}\right)+1}(f)(x) \geq \frac{C_{\delta}}{N} c_{0}^{\prime} h^{-N d} \int_{y \in \mathcal{O}_{N, \epsilon}, \operatorname{dist}(y, x) \leq c_{1}^{\prime} h / 2} f(y) d y
\end{aligned}
$$

By induction on $n$, we thus get

$$
\begin{aligned}
& \operatorname{dist}\left(x, x^{0}\right) \leq 2 \alpha\left(x^{0}\right)-c_{1}^{\prime} h \Longrightarrow \\
& K_{h, N}^{M\left(N, x^{0}\right)+n}(f)(x) \geq\left(\frac{C_{\delta}}{N}\right)^{n} c_{0}^{\prime} h^{-N d} \int_{y \in \mathcal{O}_{N, \epsilon}, \operatorname{dist}(y, x) \leq c_{1}^{\prime} \frac{h}{2^{n}}} f(y) d y
\end{aligned}
$$

Since $n$ is bounded, we get the desired result with $h_{0}=\min \left(\min _{x^{0} \in F} \alpha_{x^{0}} / c_{1}^{\prime}, h_{0}^{\prime}\right)$.

To complete the progf, let us show ( 9.32 ) by induction on $N$. The cas $N=1$ is obvious. Suppose that ( $\left(\frac{65}{4.32}\right)$ holds for $N-1$ discs. Let $x^{0} \in \overline{\mathcal{O}}_{N, \epsilon}$ being fixed. Thanks to Lemma $\frac{1 \text { em }}{1.2 \text {, we }}$ can suppose that there exists an open neighbourhood $U$ of $x^{0}$ a direction $\nu \in S^{d-1}$ and $\delta>0$ such that (?!? ) holds with $j=1$. Let us denote $x=\left(x_{1}, x^{\prime}\right)$ and

$$
K_{h, N}=K_{h, N, 1}+K_{h, N,>}
$$

with

$$
K_{h, N, 1} f(x)=\frac{h^{-d}}{N} \int_{\left(y_{1}, x^{\prime}\right) \in \mathcal{O}_{N, \epsilon}} \varphi\left(\frac{x_{1}-y_{1}}{h}\right) f\left(y_{1}, x^{\prime}\right) d y_{1} .
$$

We also denote $G(\nu, \delta)=\Gamma_{+}(\nu, \delta) \cap\left\{\left|x_{1}\right|>\frac{\delta}{2}\right\}$. Then, we have the following

sublem7bis Lemma 4.6 For any $\left.\left.\delta^{\prime} \in\right] 0, \delta / 2\right]$, there exists $C>0, \alpha>0, h_{0}>0$ and $r_{0}>0$ such $\left.\left.\left.\forall r \in] 0, r_{0}\right], \forall h \in\right] 0, h_{0}\right], \forall x \in U \cap \mathcal{O}_{N, \epsilon}, \forall \tilde{x} \in x+h\left(G\left(\nu, \delta^{\prime}\right) \times B(0, r)^{N-1}\right)$ with $\tilde{x}^{\prime} \in \mathcal{O}_{N-1, \epsilon}$, we have $\tilde{x} \in \mathcal{O}_{N, \epsilon}$ and

$$
K_{h, N,>} f(\tilde{x}) \geq C K_{\alpha h, N-1}\left(f\left(\tilde{x}_{1}, .\right)\right)\left(\tilde{x}^{\prime}\right)
$$

for any non-negative function $f$. In particular, for all $M \in \mathbb{N}^{*}$, there exists $C, r_{0}, h_{0}, a$ as above such that $\forall x \in U \cap \mathcal{O}_{N, \epsilon}, \forall \tilde{x} \in x+h\left(G\left(\nu, \delta^{\prime}\right) \times B(0, r)^{N-1}\right)$, we have

$$
K_{h, N,>}^{M} f(\tilde{x}) \geq C K_{\alpha h, N-1}^{M}\left(f\left(\tilde{x}_{1}, .\right)\right)\left(\tilde{x}^{\prime}\right)
$$


Proof Inequality $\left(\frac{e q: \mathrm{m} 2}{4.39}\right)$ is obtained easily from $\left(\frac{\mathrm{eq}: \mathrm{m} 1}{4.38}\right.$ by induction on $M$. To prove (4.38), we observe that for non-negative $f$ and $\alpha \in] 0,1[$ we have

$$
K_{h, N,>} f(\tilde{x}) \geq \frac{h^{-d}}{N} \sum_{j=2}^{N} \int_{A_{j, \alpha, h}(\tilde{x})} f\left(\tilde{x}_{1}, \ldots, y_{j}, \ldots, \tilde{x}_{N}\right) d y_{j}
$$

with $A_{j, \alpha, h}(\tilde{x})=\left\{z \in \Omega,\left|\tilde{x}_{j}-z\right|<\alpha h\right.$ and $\left.\forall k \neq j,\left|\tilde{x}_{k}-z\right|>\epsilon\right\}$. Let $B_{j, \alpha, h}(\tilde{x})=\{z \in$ $\Omega,\left|\tilde{x}_{j}-z\right|<\alpha h$ and $\left.\forall k \neq 1, j,\left|\tilde{x}_{k}-z\right|>\epsilon\right\}$. Then $A_{j, \alpha, h} \subset B_{j, \alpha, h}$ and we claim that for $\alpha, r>0$ small enough and $\tilde{x} \in x+h\left(G\left(\nu, \delta^{\prime}\right) \times B(0, r)^{N-1}\right)$ with $\tilde{x}^{\prime} \in \mathcal{O}_{N-1, \epsilon}$, we have $B_{j, \alpha, h}(\tilde{x})=A_{j, \alpha, h}(\tilde{x})$. Indeed, let $\tilde{x}_{1}=x_{1}+h u_{1}$ with $u_{1} \in G\left(\nu, \delta^{\prime}\right)$ and $\tilde{x}^{\prime} \in \mathcal{O}_{N-1, \epsilon}$ be such that $\left|\tilde{x}_{j}-x_{j}\right|<h r$. Then for $z \in B_{j, \alpha, h}(\tilde{x})$ we have

$$
\left|\tilde{x}_{1}-z\right|=\left|x_{1}-x_{j}+h v_{1}\right|
$$

with $v_{1}=u_{1}+\frac{x_{j}-\tilde{x}_{j}}{h}+\frac{\tilde{x}_{j}-z}{h}$. Taking $\alpha, r$ small enough (w.r.t. $\delta$ ) it follows that $v_{1} \in$ $\Gamma_{+}(\nu, \delta)$. Consequently, Lemma $\frac{1}{4.2}$ shows that $\left|\tilde{x}_{1}-z\right|>\epsilon$ and hence $z \in A_{j, \alpha, h}(\tilde{x})$ (the same argument shows that $\left.\tilde{x} \in \mathcal{O}_{N, \epsilon}\right)$. Therefore,

$$
K_{h, N,>} f(\tilde{x}) \geq \frac{h^{-d}}{N} \sum_{j=2}^{N} \int_{B_{j, \alpha, h}(\tilde{x})} f\left(\tilde{x}_{1}, \ldots, y_{j}, \ldots, \tilde{x}_{N}\right) d y_{j}=\frac{N}{N-1} K_{\alpha h, N-1}\left(f\left(\tilde{x}_{1}, .\right)\right)\left(\tilde{x}^{\prime}\right)
$$

and the proof of Lemma $\frac{\text { sublem7bis }}{4.6 \text { is complete. }}$

Using this Lemma we can complete the proof of $\left(\frac{95}{4.32}\right)$. Let $\left.\left.p \in \mathbb{N}, \alpha \in\right] 0, \alpha_{0}\right]$ and $x \in \mathcal{O}_{N, \epsilon}$, then

$$
\begin{aligned}
K_{h, N}^{p+1} f(x) & \geq K_{h, N, 1} K_{h, N,>}^{p} f(x) \\
& \geq \frac{h^{-d}}{N} \int_{\left(z_{1}, x^{\prime}\right) \in \mathcal{O}_{N, \epsilon}, z_{1} \in x_{1}+h G\left(\nu, \delta^{\prime}\right)} K_{h, N,>}^{p} f\left(z_{1}, x^{\prime}\right) d z_{1} \\
& \geq C \frac{h^{-d}}{N} \int_{\left(z_{1}, x^{\prime}\right) \in \mathcal{O}_{N, \epsilon}, z_{1} \in x_{1}+h G\left(\nu, \delta^{\prime}\right)} K_{\alpha h, N-1}^{p}\left(f\left(z_{1}, .\right)\right)\left(x^{\prime}\right) d z_{1}
\end{aligned}
$$

thanks to Lemma $\frac{\text { sublem7bis }}{4.6 \text {. From }}$ the induction hypothesis we can choose $p \in \mathbb{N}$ so that

$$
K_{h, N}^{p+1} f(x) \geq C h^{-N d} \int_{\left(z_{1}, x^{\prime}\right) \in \mathcal{O}_{N, \epsilon}, z_{1} \in x_{1}+h G(\nu, \delta)} \int_{\left|x^{\prime}-y^{\prime}\right|<\alpha h, y^{\prime} \in \mathcal{O}_{N-1, \epsilon}} f\left(z_{1}, y^{\prime}\right) d y^{\prime} d z_{1}
$$

Hence, for any $\beta \in] 0,1]$ we get

$$
K_{h, N}^{p+2} f(x) \geq K_{h, N}^{p+1} K_{h, N, 1} f(x) \geq C h^{-N d} \int_{D_{\alpha, \beta, h}(x)} f\left(y_{1}, y^{\prime}\right) \gamma_{h}\left(x, y_{1}\right) d y d y^{\prime}
$$

with

$$
D_{\alpha, \beta, h}(x)=\left\{y \in \mathcal{O}_{N, \epsilon},\left|x^{\prime}-y^{\prime}\right|<\alpha h,\left|x_{1}-y_{1}\right|<\beta h\right\}
$$

and

$$
\gamma_{h}\left(x, y_{1}\right)=h^{-d} \int_{\left(z_{1}, x^{\prime}\right) \in \mathcal{O}_{N, \epsilon}, z_{1} \in x_{1}+h G\left(\nu, \delta^{\prime}\right)} 1_{\left|z_{1}-y_{1}\right|<h} d z_{1}
$$


We have to show that $\gamma_{h}$ is bounded from below by a positive constant, uniformly with respect to $\left(x, y_{1}\right)$. For this purpose, we observe that for $y \in D_{\alpha, \beta, h}(x)$ and $\beta, \delta^{\prime}$ small enough we have

$$
\gamma_{h}\left(x, y_{1}\right) \geq C h^{-d} \int_{\left(z_{1}, x^{\prime}\right) \in \mathcal{O}_{N, \epsilon}, z_{1} \in x_{1}+h G\left(\nu, \delta^{\prime}\right)} d z_{1}=\int_{|u|<\alpha, u \in G\left(\nu, \delta^{\prime}\right)} 1_{\left(x_{1}+h u, x^{\prime}\right) \in \mathcal{O}_{N, \epsilon}} d u
$$

Using again Lemma $\frac{1 \text { em7ter }}{1.2, \text { we get }}$

$$
\gamma_{h}\left(x, y_{1}\right) \geq C \int_{|u|<\alpha, u \in G\left(\nu, \delta^{\prime}\right)} d u=C_{0}>0 .
$$

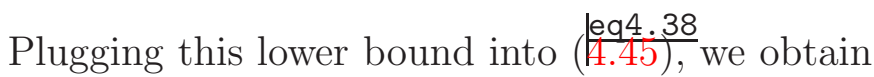

$$
K_{h, N}^{p+2} \geq C h^{-N d} \int_{D_{\alpha, \beta, h}(x)} f(y) d y
$$

and the proof of $\left(\frac{95}{4.32}\right)$ is complete. This achieves the proof of lemma $\frac{1 \mathrm{em} 7 \mathrm{bis}}{4.5 .}$

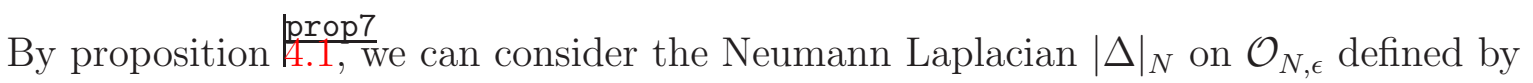

$$
|\Delta|_{N}=-\frac{\alpha_{d}}{2 N} \Delta, D\left(|\Delta|_{N}\right)=\left\{u \in H^{1}\left(\mathcal{O}_{N, \epsilon}\right),-\Delta u \in L^{2}\left(\mathcal{O}_{N, \epsilon}\right),\left.\partial_{n} u\right|_{\partial \mathcal{O}_{N, \epsilon}}=0\right\}
$$

We still denote $0=\nu_{0}<\nu_{1}<\nu_{2}<\ldots$ the spectrum of $|\Delta|_{N}$ and $m_{j}$ the multiplicity of $\nu_{j}$. Our main result is the following.

thm3 Theorem 4.1 Let $N \geq 2$ be fixed. Let $\epsilon>0$ be small enough such that proposition $\frac{\text { prop7 }}{4.1}$

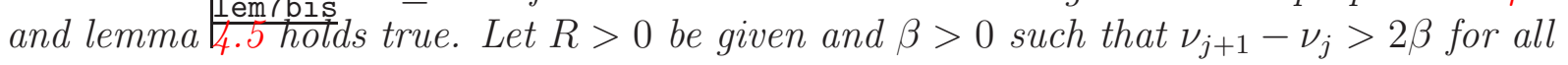
$j$ such that $\nu_{j+2} \leq R$.

There exists $\left.h_{0}>0, \delta_{0} \in\right] 0,1 / 2\left[\right.$ and constants $C_{i}>0$ such that for any $\left.\left.h \in\right] 0, h_{0}\right]$, the following holds true.

i) The spectrum of $T_{h}$ is a subset of $\left[-1+\delta_{0}, 1\right], 1$ is a simple eigenvalue of $T_{h}$, and $\operatorname{Spec}\left(T_{h}\right) \cap\left[1-\delta_{0}, 1\right]$ is discrete. Moreover,

$$
\begin{aligned}
& \left.\left.\operatorname{Spec}\left(\frac{1-T_{h}}{h^{2}}\right) \cap\right] 0, R\right] \subset \cup_{j \geq 1}\left[\nu_{j}-\beta, \nu_{j}+\beta\right] \\
& \sharp \operatorname{Spec}\left(\frac{1-T_{h}}{h^{2}}\right) \cap\left[\nu_{j}-\beta, \nu_{j}+\beta\right]=m_{j} \quad \forall \nu_{j} \leq R
\end{aligned}
$$

and for any $0 \leq \lambda \leq \delta_{0} h^{-2}$, the number of eigenvalues of $T_{h}$ in $\left[1-h^{2} \lambda, 1\right]$ (with multiplicity) is bounded by $C_{1}(1+\lambda)^{d N / 2}$.

ii) The spectral gap $g(h)$ satisfies

$$
\lim _{h \rightarrow 0^{+}} h^{-2} g(h)=\nu_{1}
$$

and the following estimate holds true for all integer $n$

$$
\sup _{x \in \mathcal{O}_{N, \epsilon}}\left\|T_{h}^{n}(x, d y)-\frac{d y}{\operatorname{Vol}\left(\mathcal{O}_{N, \epsilon}\right)}\right\|_{T V} \leq C_{4} e^{-n g(h)}
$$


The rest of this section is devoted to the proof of theorem $\frac{\operatorname{thm} 3}{4.1 .}$

Let $\mu_{h}(x, d y)$ be given by $\frac{\text { eq } 4.5}{1.30}$ and $\mu_{h}(f)(x)=\int_{\mathcal{O}_{N, \epsilon}} f(y) \mu_{h}(x, d y)$. Thanks to the positivity of $\mu_{h}(x, d y)$, using the Markov property of $T_{h}^{M}$ and Lipschitz-continuity of the boundary, we get for some $\delta_{0}^{\prime}>0$ independant on $h>0$ small enough

$$
\left\|\mu_{h}\right\|_{L^{\infty}, L^{\infty}} \leq 1-\inf _{x \in \mathcal{O}_{N, \epsilon}} \int_{\mathcal{O}_{N, \epsilon}} c_{0} h^{-N d} \varphi_{N d}\left(\frac{x-y}{c_{1} h}\right) d y<1-\delta_{0}^{\prime}
$$

Since by $\frac{\text { eq } 4.5}{4.30} \mu_{h}$ is selfadjoint on $L^{2}\left(\mathcal{O}_{N, \epsilon}\right)$, we get also

$$
\left\|\mu_{h}\right\|_{L^{1}, L^{1}} \leq 1-\delta_{0}^{\prime}
$$

and by interpolation it follows that $\left\|\mu_{h}\right\|_{L^{2}, L^{2}} \leq 1-\delta_{0}^{\prime}$. In particular the essential spectrum of $T_{h}^{M}$ is contained in $\left[0,1-\delta_{0}^{\prime}\right]$ so that $\sigma_{e s s}\left(T_{h}\right) \subset\left[0,1-2 \delta_{0}\right]$ with $2 \delta_{0}=1-\left(1-\delta_{0}^{\prime}\right)^{1 / M}$. Thus $\operatorname{Spec}\left(T_{h}\right) \cap\left[1-\delta_{0}, 1\right]$ is discrete. Let us verify that decreasing $\delta_{0}>0$, we may also assume

$$
\operatorname{Spec}\left(T_{h}\right) \subset\left[-1+\delta_{0}, 1\right] .
$$

Thanks to the Markov property of $T_{h}^{M}$, to prove this, it suffices to find $M \in 2 \mathbb{N}+1$ such that

$$
\int_{\Omega} \int_{\Omega}(u(x)+u(y))^{2} T_{h}^{M}(x, d y) d x \geq \delta_{0}\|u\|_{L^{2}}^{2}
$$

for any $u \in L^{2}(\Omega)$. Moreover, thanks to the proof of Lemma lem7bis that for any $n \in \mathbb{N}$,

$$
\int_{\Omega} \int_{\Omega}(u(x)+u(y))^{2} T_{h}^{M+n}(x, d y) d x \geq c_{0}(n) h^{-N d} \int_{\Omega \times \Omega}(u(x)+u(y))^{2} \varphi_{N d}\left(\frac{x-y}{c_{1}(n) h}\right) d x d y .
$$

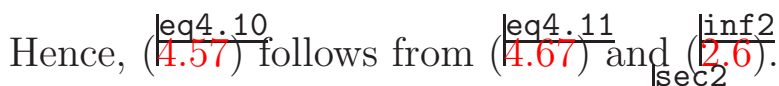

Following the strategy of section $\frac{5 \text { we }}{2 \text { we }} \mathcal{O}_{N, \epsilon}$ in a large box $\left.B=\right]-A / 2, A / 2\left[^{N d}\right.$ and

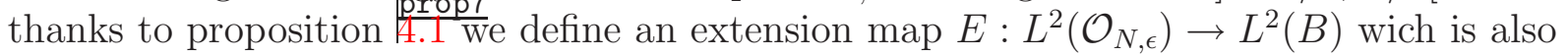
bounded from $H^{1}\left(\mathcal{O}_{N, \epsilon}\right)$ into $H^{1}(B)$. We denote

$$
\mathcal{E}_{h, k}(u)=\left\langle\left(1-T_{h}^{k}\right) u, u\right\rangle_{L^{2}\left(\mathcal{O}_{N, \epsilon}\right)}
$$

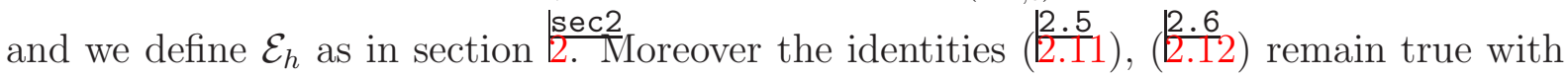
obvious modifications.

lem8 Lemma 4.7 There exist $C_{0}, h_{0}>0$ such that the following holds true for any $\left.\left.h \in\right] 0, h_{0}\right]$ and any $u \in L^{2}\left(\mathcal{O}_{N, \epsilon}\right)$

Proof. Thanks to Lemma $\frac{\operatorname{lem} 1}{2.2 \text { we have }}$

$$
\mathcal{E}_{h}(E(u)) \leq C_{0}\left(\mathcal{E}_{h, M}(u)+h^{2}\|u\|_{L^{2}}^{2}\right)
$$

$$
\mathcal{E}_{h}(E(u)) \leq C_{0}\left(\int_{\mathcal{O}_{N, \epsilon} \times \mathcal{O}_{N, \epsilon}}(u(x)-u(y))^{2} c_{0} h^{-N d} \varphi_{N d}\left(\frac{x-y}{c_{1} h}\right) d y d x+h^{2}\|u\|_{L^{2}\left(\mathcal{O}_{N, \epsilon}\right)}\right)
$$

Combined with $\left(\frac{\mathrm{eq} 4.5}{4.30}\right)^{2}$, this shows that

$$
\mathcal{E}_{h}(E(u)) \leq C_{0}\left(\int_{\mathcal{O}_{N, \epsilon} \times \mathcal{O}_{N, \epsilon}}(u(x)-u(y))^{2} T_{h}^{M}(x, d y) d x+h^{2}\|u\|_{L^{2}\left(\mathcal{O}_{N, \epsilon}\right)}\right)
$$

and the proof is complete. 
lem9 Lemma 4.8 For any $0 \leq \lambda \leq \delta_{0} / h^{2}$, the number of eigenvalues of $T_{h}$ in $\left[1-h^{2} \lambda, 1\right]$ (with multiplicity) is bounded by $C_{1}(1+\lambda)^{N d / 2}$. Moreover, any eigenfuntion $T_{h}(u)=\lambda u$ with $\left.\lambda \in] 1-\delta_{0}, 1\right]$ satisfies the bound

$$
\|u\|_{L^{\infty}} \leq C_{2} h^{-N d / 2}\|u\|_{L^{2}}
$$

Proof $_{5}$ Suppose that $T_{h}(u)=\lambda u$ with $\lambda \in\left[1-\delta_{0}, 1\right]$, then $T_{h}^{M} u=\lambda^{M} u$ and thanks to $\left(\frac{29}{4.30}\right)$, we get

$$
\left\|\left(\mu_{h}-\lambda^{M}\right) u\right\|_{L^{\infty}}=O\left(h^{-N d / 2}\right)
$$

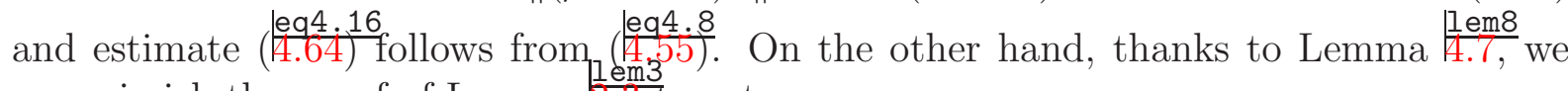
can mimick the proof of Lemma $\frac{1 \mathrm{em} 3}{2.3 \text { to get }}$

$$
\zeta_{M}(\lambda, h) \leq C(1+\lambda)^{N d / 2}
$$

Let $\zeta_{k}(\lambda, h)$ be the number of eigenvalues of $T_{h}^{k}$ in the interval $\left[1-h^{2} \lambda, 1\right]$ for $h^{2} \lambda<\delta_{0}$. Then from $\frac{1.5 \%}{4 .}$ one has

$$
\zeta_{1}(\lambda, h)=\zeta_{k}\left(\frac{1-\left(1-h^{2} \lambda\right)^{k}}{h^{2}}, h\right)
$$

Combining $\left(\frac{\mathrm{eq} 4.18}{4.66)}\right.$ and $\left(\frac{\mathrm{eg} 4.11}{4.6 \%}\right)$ we get easily the anounced estimate. The proof of lemma 4.8 is complete.

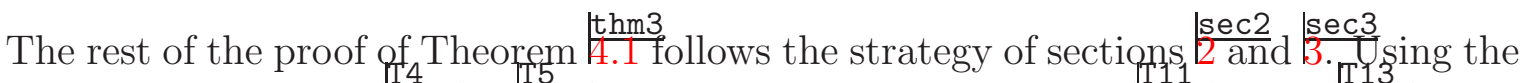
spectral decomposition $\left(\frac{\mathbb{1} 4}{2.39}\right)$, $\left(\frac{\mathrm{TS}}{2.40}\right)$ we get easily the estimates $\left(\frac{T 11}{2.46}\right)$ and $\left(\frac{T 13}{2.48}\right)$, and it remains to estimate $T_{h, 1}^{n}$. Following the proof of Lemma $\frac{1 e m 4}{2.4}$ we can find $\alpha>0$ small enough and $C>0$ such that the following Nash inequality holds with $1 / D=2-4 / p>0$

$$
\|u\|_{L^{2}}^{2+1 / D} \leq C h^{-2}\left(\left(\mathcal{E}_{h, M}(u)+h^{2}\|u\|_{L^{2}}^{2}\right)\|u\|_{L^{1}}^{1 / D}, \quad \forall u \in E_{\alpha}\right.
$$

From this inequality, we deduce that for $k \geq h^{-2}$,

$$
\left\|T_{1, h}^{k M}\right\|_{L^{\infty}, L^{\infty}} \leq C e^{-k M g(h)} .
$$

and this implies for $k \geq h^{-2}$, since the contributions of $T_{2, h}^{k M}, T_{3, h}^{k M}$ are neglectible,

$$
\left\|T_{h}^{k M}\right\|_{L^{\infty}, L^{\infty}} \leq C^{\prime} e^{-k M g(h)} .
$$

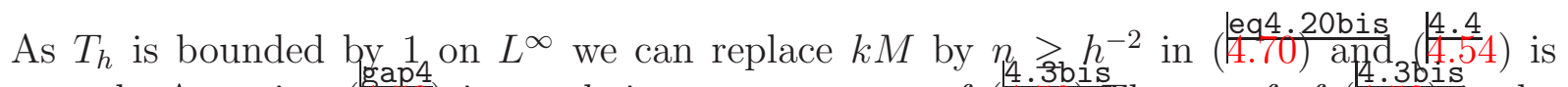

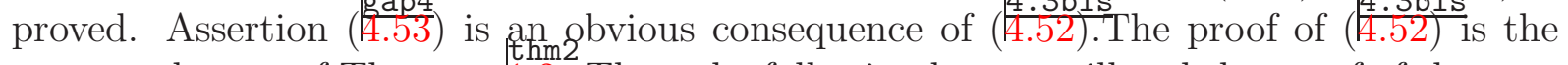
same as the one of Theorem $\frac{\operatorname{thm} 2}{1.2 .}$ Thus, the following lemma will end the proof of theorem thm3

lem10 Lemma 4.9 Let $\theta \in C^{\infty}\left(\overline{\mathcal{O}}_{N, \epsilon}\right)$ be such that $\operatorname{supp}(\theta) \cap \Gamma_{\text {sing }}=\emptyset$ and $\left.\partial_{n} \theta\right|_{\Gamma_{\text {reg }}}=0$. Then

$$
\left(1-T_{h}\right) \theta=h^{2}|\Delta|_{N} \theta+r, \quad\|r\|_{L^{2}}=O\left(h^{5 / 2}\right) .
$$

Proof. Let $\theta \in C^{\infty}\left(\overline{\mathcal{O}}_{N, \epsilon}\right)$ be such that $\operatorname{supp}(\theta) \cap \Gamma_{\text {sing }}=\emptyset$ and $\left.\partial_{n} \theta\right|_{\Gamma_{\text {reg }}}=0$ and denote $Q_{h}=1-T_{h}$. Then $Q_{h}=\frac{1}{N} \sum_{j=1}^{N} Q_{j, h}$ with

$$
Q_{j, h} \theta(x)=\frac{h^{-d}}{\operatorname{Vol}\left(B_{1}\right)} \int_{\Omega} 1_{\left|x_{j}-y\right|<h} \Pi_{k \neq j} 1_{\left|x_{k}-y\right|>\epsilon}\left(f(x)-f\left(\pi_{j}(x)+\sigma_{j}(y)\right)\right) d y
$$




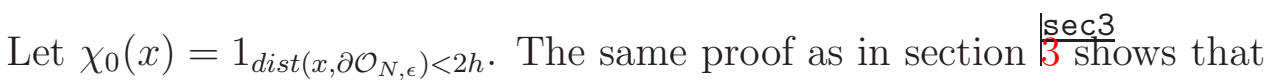

$$
\left(1-\chi_{0}\right) Q_{j, h} \theta(x)=-\frac{\alpha_{d}}{2} h^{2} \partial_{j}^{2} \theta(x)+O_{L^{\infty}}\left(h^{3}\right)
$$

so that

$$
\left(1-\chi_{0}\right) Q_{h} \theta(x)=h^{2}|\Delta|_{N} \theta(x)+O_{L^{2}}\left(h^{3}\right) .
$$

We study $\chi_{0} Q_{h} \theta$. As $\left\|\chi_{0}\right\|_{L^{2}}=O\left(h^{1 / 2}\right)$ it suffices to show that $\left\|\chi_{0} Q_{h} \theta\right\|_{L^{\infty}}=O\left(h^{2}\right)$. On the other hand, by Taylor expansion, we have

$$
\chi_{0} Q_{j, h} \theta(x)=-\frac{h \chi_{0}(x)}{\operatorname{Vol}\left(B_{1}\right)} \int_{|z|<1} \Pi_{k \neq j} 1_{\left|x_{j}+h z-x_{k}\right|>\epsilon} 1_{x_{j}+h z \in \Omega} z . \partial_{j} \theta(x) d y_{j}+O_{L^{\infty}}\left(h^{2}\right)
$$

Hence, it suffices to show that

$$
v(x)=\chi_{0}(x) \sum_{j=1}^{N} \int_{|z|<1} \Pi_{k \neq j} 1_{\left|x_{j}+h z-x_{k}\right|>\epsilon} 1_{x_{j}+h z \in \Omega} z . \partial_{j} \theta(x) d y_{j}
$$

satisfies $\|v\|_{L^{\infty}}=O(h)$. Since $\operatorname{dist}\left(\operatorname{support}(\theta), \Gamma_{\text {sing }}\right)>0$, there exists disjoints compact sets $F_{l} \subset \Gamma_{r e g, e x t, l}$, and $F_{i, j} \subset \Gamma_{\text {reg,int },(i, j)}$ such that

$$
\operatorname{support}(\theta) \subset \cup_{l}\left\{x, \operatorname{dist}\left(x, F_{l}\right) \leq 4 h\right\} \cup_{i, j}\left\{x, \operatorname{dist}\left(x, F_{i, j}\right) \leq 4 h\right\}
$$

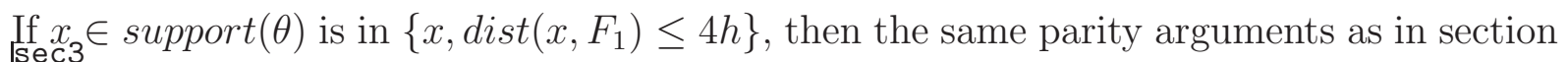
3 show that

$$
v(x)=\chi_{0}(x) \int_{|z|<1, x_{1}+h z \in \Omega} z \cdot \partial_{1} \theta(x) d z=O(h)
$$

If $x \in \operatorname{support}(\theta)$ is in $\left\{x, \operatorname{dist}\left(x, F_{1,2}\right) \leq 4 h\right\}$, then

$$
v(x)=\chi_{0}(x) \int_{|z|<1} z \cdot\left(\partial_{1} \theta(x) 1_{\left|x_{1}+h z-x_{2}\right|>\epsilon}+\partial_{2} \theta(x) 1_{\left|x_{2}+h z-x_{1}\right|>\epsilon}\right) d z
$$

and the result follows from $\left(x_{1}-x_{2}\right) \cdot\left(\partial_{1} \theta-\partial_{2} \theta\right)(x)=0(h)$ for $\left\{x\right.$, fist $\left.\left(x, F_{1,2}\right) \leq 4 h\right\}$, since $\partial_{n} \theta$ vanish on the boundary $\left|x_{1}-x_{2}\right|=\epsilon$. The proof of lemma $\frac{1}{4.9}$ is complete.

\section{References}

[DL07] P. Diaconis and G. Lebeau. Microlocal analysis for the metropolis algorithm. submitted, 2007. 5

[MRR $\left.{ }^{+} 53\right]$ N. Metropolis, A. Rosenbluth, M. Rosenbluth, A. Teller, and E. Teller. Equations of state calculations by fast computing machines. J. Chem. Phys., 21:1087-1092, 1953. 2 